\title{
Neuropilin-1 upregulation elicits adaptive resistance to oncogene-targeted therapies
}

\author{
Sabrina Rizzolio, ${ }^{1,2}$ Gabriella Cagnoni, ${ }^{1,2}$ Chiara Battistini, ${ }^{1,2}$ Stefano Bonelli, ${ }^{3,4}$ Claudio Isella, ${ }^{1,2}$ Jo A. Van Ginderachter, ${ }^{3,4}$ \\ René Bernards, ${ }^{5}$ Federica Di Nicolantonio, ${ }^{1,2}$ Silvia Giordano, ${ }^{1,2}$ and Luca Tamagnone ${ }^{1,2}$ \\ 'Candiolo Cancer Institute-FPO, IRCCS, Candiolo, Italy. ${ }^{2}$ Oncology Department, University of Torino, Turin, Italy. ${ }^{3}$ Myeloid Cell Immunology Lab, VIB Center for Inflammation Research, Brussels, Belgium. \\ ${ }^{4}$ Lab of Cellular and Molecular Immunology, Vrije Universiteit Brussel, Brussels, Belgium. ${ }^{5}$ Division of Molecular Carcinogenesis, Oncode Institute, The Netherlands Cancer Institute, Amsterdam, Netherlands.
}

\begin{abstract}
Cancer cell dependence on activated oncogenes is therapeutically targeted, but acquired resistance is virtually unavoidable. Here we show that the treatment of addicted melanoma cells with BRAF inhibitors, and of breast cancer cells with HER2targeted drugs, led to an adaptive rise in neuropilin-1 (NRP1) expression, which is crucial for the onset of acquired resistance to therapy. Moreover, NRP1 levels dictated the efficacy of MET oncogene inhibitors in addicted stomach and lung carcinoma cells. Mechanistically, NRP1 induced a JNK-dependent signaling cascade leading to the upregulation of alternative effector kinases ECFR or IGF1R, which in turn sustained cancer cell growth and mediated acquired resistance to BRAF, HER2, or MET inhibitors. Notably, the combination with NRP1-interfering molecules improved the efficacy of oncogene-targeted drugs and prevented or even reversed the onset of resistance in cancer cells and tumor models. Our study provides the rationale for targeting the NRP1-dependent upregulation of tyrosine kinases, which are responsible for loss of responsiveness to oncogene-targeted therapies.
\end{abstract}

\section{Introduction}

Precision oncology relies on treating individual tumors with drugs that target their specific vulnerabilities. Cancer cells are often characterized by the constitutive activation of an oncogenic signaling cascade to which they become dependent (oncogene addiction), a liability that can be exploited for therapy (1). For example, half of all melanomas are characterized by oncogenic BRAF mutations, which render them susceptible to treatment with BRAF inhibitors. Yet, subsets of tumor cells may carry intrinsic (primary) resistance or initially drug-sensitive cells may subsequently develop refractoriness to oncogene inhibitors (acquired resistance), thereby nullifying the efficacy of targeted therapy (2-4). Thus, major challenges are the identification of tumor traits that predict drug susceptibility and the development of novel therapeutic approaches to prevent or delay the onset of drug resistance by disabling underlying molecular mechanisms. Concomitant genetic changes found in tumors may be responsible for primary refractoriness to targeted therapies or - when present at the subclonal level - may lead to outgrowth of drug-resistant tumors over time. However, targeted therapies often cease to be effective due to adaptive mechanisms triggered in response to treatment (5). For instance, resistance to oncogene-targeted drugs is frequently due to the upregulation of parallel signaling cascades, which can sustain cancer cell viability and proliferation despite the blunting of the pathway to which the tumor is addicted. In particular, it has been shown that cancer cells can exploit the presence of cytokines

Authorship note: CC and CB contributed equally to this work. Conflict of interest: The authors have declared that no conflict of interest exists. Submitted: December 13, 2017; Accepted: June 22, 2018. Reference information: / Clin Invest. 2018;128(9):3976-3990. https://doi.org/10.1172/JCI99257. and growth factors in the tumor microenvironment by increasing the expression and/or activity of tyrosine kinase receptors, such as EGFR, HGFR, FGFR, and others (6). Although these changes may be reversible in principle, they nonetheless jeopardize the clinical efficacy of targeted inhibitors, leading to progressive disease.

Neuropilin-1 (NRP1) is a transmembrane protein overexpressed in advanced human tumors, typically showing growthpromoting functions in cancer cells $(7,8)$. NRP1 was initially described as a coreceptor for secreted semaphorins and for vascular endothelial growth factors (VEGFs), in association with plexins or VEGF receptors, respectively. However, we have shown that NRP1 also interacts with epidermal growth factor receptor (EGFR) at the cell surface, promoting ligand-induced EGFR clustering and intracellular signaling (9). Additional growth factor-induced signaling cascades have been associated with NRP1, though the implicated molecular mechanisms remain largely unclear (10). Thus, NRP1 seems to act as a receptor hub on the cell surface, promoting multiple signaling cascades $(11,12)$.

The mechanisms modulating NRP1 expression in cancer cells are still controversial. For instance, NRP1 may be transcriptionally induced by growth factors and by the activation of the RAS-MAPK signaling pathway (13-15); on the other hand, NRP1 transcripts are proposed targets of miRNA-338 (16) and other miRNAs. Notably, NRP1 is widely expressed in carcinoma cells (although at different levels), whereas it is hardly present in neural crest derivatives, including melanocytes and melanoma cells.

Previous studies support the notion that elevated NRP1 expression in tumors correlates with poor outcome $(7,12)$; however, the underlying mechanisms have not been elucidated. In the present study, we explore the hypothesis that NRP1 expression confers a growth advantage to oncogene-addicted cancer cells treated with targeted inhibitors, thus contributing to drug resistance. We 
investigated melanoma cells characterized by $B R A F$-activating mutations and oncogenic addiction, as well as several carcinoma lines derived from stomach, lung, or breast cancer carrying MET or HER2 oncogene amplification and constitutive signaling. Our data reveal a novel role for NRP1 in controlling the therapeutic response to targeted oncogene inhibitors, and identify NRP1 as a novel target for therapy to fight drug resistance.

\section{Results}

$B R A F$-inhibitor resistance in melanoma cells is dependent on NRP1 de novo expression, associated with the downregulation of the SOX1Oeffector miRNA-338. As a prototypical example of oncogenic addiction, approximately half of melanomas carry a constitutively activated BRAF kinase, whereby the treatment with targeted inhibitors initially achieves remarkable therapeutic success. Unfortunately, drug resistance often ensues, dependent on the upregulation of alternative signaling pathways (3). For instance, we have previously shown that BRAF-addicted melanoma cells, upon treatment with targeted inhibitors, undergo adaptive gene expression reprogramming and develop drug resistance associated with the downregulation of the transcription factor SOX10 (17), a known marker of neural crest lineage differentiation. This was associated with the upregulation of the EGFR tyrosine kinase, as well as of other growth factor receptor signaling cascades such as TGFBR2 and PDGFRB. Yet, the pathway responsible for these adaptive changes has not been fully elucidated. Intriguingly, we and others have demonstrated a role for NRP1 in controlling cancer cell growth by promoting signaling cascades mediated by EGFR, TGF $\beta$, PDGFR, and others (11). In fact, melanoma cells typically carry barely detectable NRP1 (see Supplemental Figure 1A; supplemental material available online with this article; https:/doi. org/10.1172/JCI99257DS1), implying that it is not basally required for their viability. However, in a genome-wide expression analysis previously performed (17), NRP1 was the third most upregulated gene in SOX10-deficient cells refractory to BRAF inhibitors, suggesting a role for NRP1 in adaptive drug resistance. We initially validated this unbiased finding by quantitative PCR (qPCR) analysis, confirming NRP1 upregulation in a range of melanoma cell lines in which SOX1O was selectively silenced by means of 2 independent shRNAs (Figure 1A and Supplemental Figure 1B). As expected, EGFR transcripts were also increased in SOX1O-silenced cells (Figure 1A and Supplemental Figure 1B). The effect was reproducible both in BRAF-mutated (A375, SK-MEL-28, and SK-MEL-5) and $B R A F$-WT melanoma cells (SK-MEL-2), indicating that it is independent of $B R A F$ oncogenic mutations and underscoring the upstream regulatory function of the SOX10 transcription factor. Expression analysis of 472 melanoma samples from The Cancer Genome Atlas (TCGA) database indicated an inverse correlation between SOX1O and NRP1 levels (Spearman's correlation coefficient: -0.542; $P<0.00001$; Supplemental Figure 1C). Moreover, there was a direct association between NRP1 and EGFR expression in the same samples (Spearman's correlation coefficient: 0.432; $P<0.00001$; Supplemental Figure 1D). We corroborated these in silico analyses by assessing NRP1, EGFR, and SOX1O expression in a panel of matched melanoma samples derived from the same patients before and after treatment with BRAF inhibitors. Indeed, we found substantial evidence of concomitant upregulation of
NRP1 and EGFR (Figure 1B). On the other hand, SOX10 was downregulated in $80 \%$ of the treated tumors, in keeping with its posited role in regulating both NRP1 and EGFR (Figure 1B).

In order to investigate the relevance of NRP1-dependent signaling in the onset of BRAF-inhibitor resistance, we established stable drug-resistant A375 and SK-MEL-28 melanoma cells by prolonged treatment in culture with escalating doses of PLX-4720 (an analogue of the clinically approved BRAF-targeted agent vemurafenib), until the surviving cells refractory to therapy displayed comparable viability to untreated parental cells (Supplemental Figure 1E). In multiple independent batches of drug-resistant cells, SOX10 mRNA levels were consistently decreased, whereas both NRP1 and EGFR were strongly upregulated at mRNA and protein levels (Figure 1, C and D and Supplemental Figure 1F). Notably, drug-resistant cell viability relied on both NRP1 and EGFR expression, whereas parental cells were not dependent on these genes (Figure 1E and Supplemental Figure 1G), suggesting the acquisition of a novel addiction pathway in melanoma cells becoming resistant to BRAF inhibitors. In order to establish the upstream mechanism driving resistance, we assayed the effect of $N R P 1$ knockdown prior to therapy and found that this was sufficient to prevent the adaptive EGFR upregulation induced by BRAF-inhibitor treatment (Figure $1 F)$. In fact, $N R P 1$ expression was required to maintain EGFR induction in SOX10-depleted and stably drug-resistant melanoma cells (Supplemental Figure 2, A and B). In a complementary approach, restoring EGFR overexpression (by cDNA transfer) in formerly drug-resistant melanoma cells subjected to NRP1 silencing was sufficient to rescue cell viability and growth in the presence of the BRAF inhibitor (Supplemental Figure 2, C and D); hence, EGFR qualifies as a major player of drug resistance downstream to NRP1. The specific functional role of NRP1 in the onset of drug resistance was also indicated by the fact that NRP1 knockdown did not impact the $\mathrm{A} 375$ cell growth rate under basal conditions (i.e., in the absence of the drug). However, after 5 weeks of culture in the presence of PLX-4720, NRP1-depleted melanoma cells were still significantly sensitive to therapy and their growth was strikingly slowed (Figure 1, G and H, and Supplemental Figure 2E), whereas control cells had developed full adaptive resistance. The relevance of NRP1 in drug resistance was ultimately demonstrated in vivo by treating mice bearing tumor xenografts of drug-resistant A375 melanoma cells, subjected to NRP1 silencing, with PLX-4720 (Figure 1I). Consistent with findings in culture, NRP1 knockdown did not impact tumor growth in vivo in the absence of the drug; however, it could successfully restore sensitivity to targeted therapy in this preclinical model of drug-resistant melanoma xenografts (Figure 1I). Altogether, these data indicate that NRP1 is induced in response to BRAF inhibitors and is a pivotal driver of resistance to BRAF-targeted therapy in melanoma. Intriguingly, we found that the NRP1 ligand VEGF was two-thirds fold induced in our drugresistant melanoma cells (not shown). Since VEGF-NRP1 signaling has been previously found to promote cancer cell growth and survival (18-22), we hypothesized that VEGF could be the trigger of this novel drug-resistance pathway; however, this doesn't seem to be the case, as there was no change in melanoma cell drug responsiveness upon VEGF silencing (Supplemental Figure 2, F and G).

We also studied the mechanism underlying the observed NRP1 transcriptional upregulation in drug-resistant SOX10- 
A

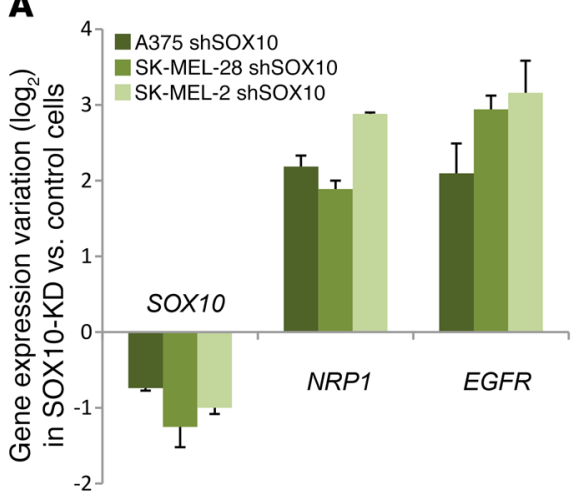

B

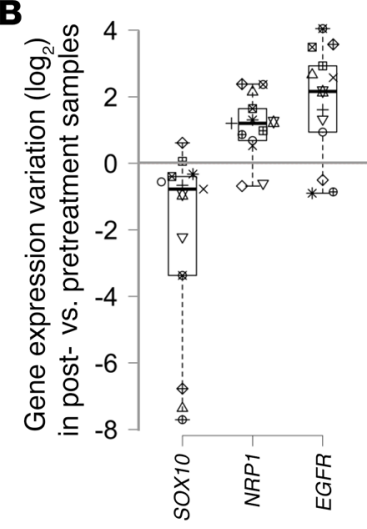

C

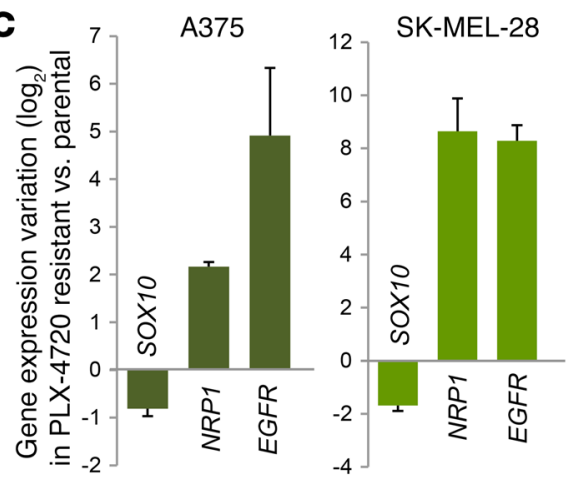

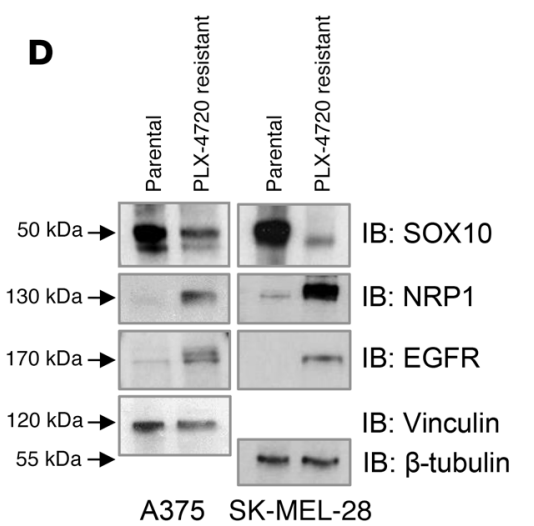

A375 SK-MEL-28
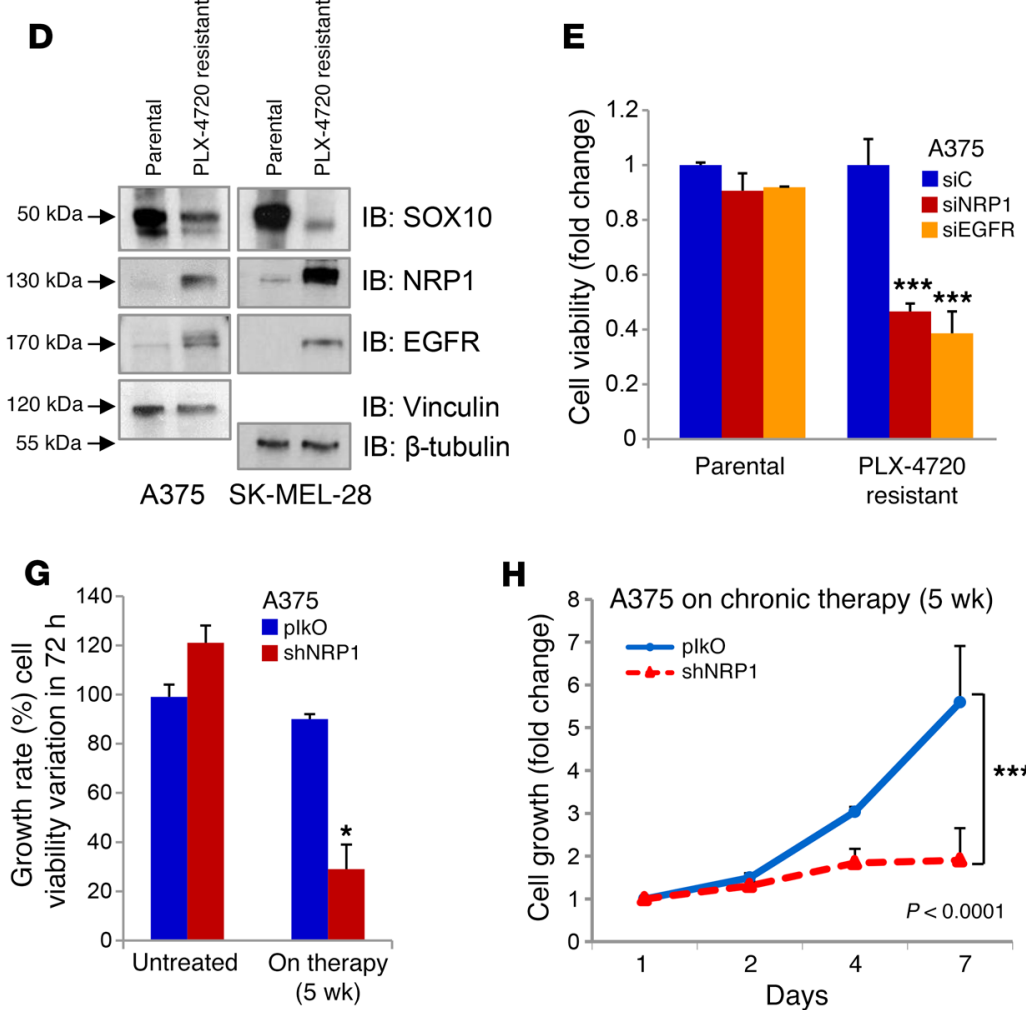

H

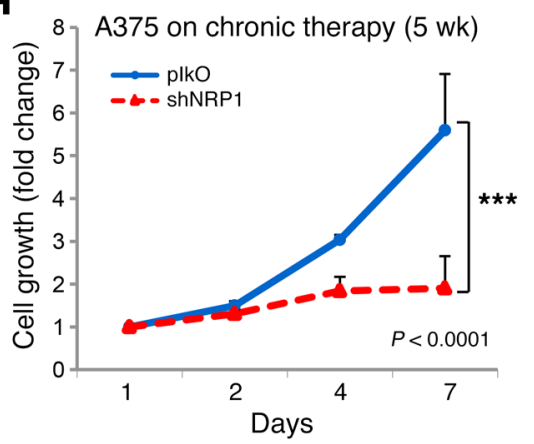

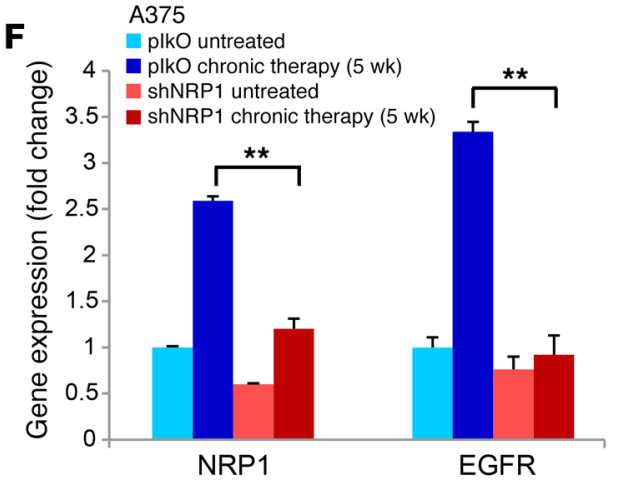

Figure 1. Adaptive NRP1 neoexpression in BRAF-addicted melanoma cells mediates acquired resistance to targeted therapy. (A) Different melanoma cells were subjected to SOX10 knockdown (KD) by inducible shRNA expression, and SOX10, NRP1, and EGFR mRNA levels were analyzed by qPCR $(n=4)$. The graph shows $\log _{2}$ fold change variations in SOX10-KD cells versus respective controls. (B) Box plot showing SOX10, NRP1, and EGFR mRNA expression variations in 12 paired melanoma samples (indicated by different symbols) obtained from the same patients before and during treatment with BRAF inhibitors ( $\log _{2}$ ratio treated/untreated). (C) qPCR analysis of SOX10, NRP1, and EGFR expression in A375 and SK-MEL-28 melanoma cells that developed acquired resistance to $2 \mu \mathrm{M}$ PLX-4720 ( $\log _{2}$ ratio drug resistant versus parental cells; $n=3$ ). (D) SOX10, NRP1, and EGFR protein expression in the same cells shown in $\mathbf{C}$; vinculin and $\beta$-tubulin levels provided loading controls (1 representative experiment of 3 repetitions; duplicate samples were run on parallel gels for staining with different antibodies). (E) The viability of parental or drug-resistant A375 cells was assessed upon NRP1 or EGFR KD with targeted siRNAs $(n=5)$. (F) A375 parental cells were subjected to NRP1 KD (by targeted shRNAs) before exposure to escalating concentrations of PLX-4720 aimed at establishing drug resistance. NRP1 and EGFR expression was analyzed after 5 weeks of treatment. The statistical analysis compared NRP1-KD samples with respective controls (carrying plkO empty vector) $(n=3)$. (G) The growth rate of control or NRP1-KD A375, either untreated or exposed to $0.5 \mu \mathrm{M}$ PLX-4720 for 5 weeks, is scored as the percentage of increase of viable cells across 72 hours $(n>3)$. (H) The growth rate of control or NRP1-KD A375 cells on therapy with $0.5 \mu$ M PLX-4720 for 5 weeks was tracked in culture over 7 days $(n>3)$. (I) Mice implanted with either mocktransduced or NRP1-KD drug-resistant A375 cells were treated daily with $20 \mathrm{mg} / \mathrm{kg}$ PLX-4720 (or vehicle only), and the tumor burden was periodically measured. The statistical analysis compared treated tumors with respective controls $(n=5$, per condition). For $\mathbf{E}, \mathbf{F}$, G, statistical significance was determined using a Student's $t$ test with Bonferroni's correction ( ${ }^{*} P<0.01$; ${ }^{* *} P<0.001$; ${ }^{* *} P<0.0001$ ); for $\mathbf{H}$ and $\mathbf{I}$, a 2-way ANOVA test with Bonferroni's correction was used $\left({ }^{* *} P<0.0001\right)$. 
A

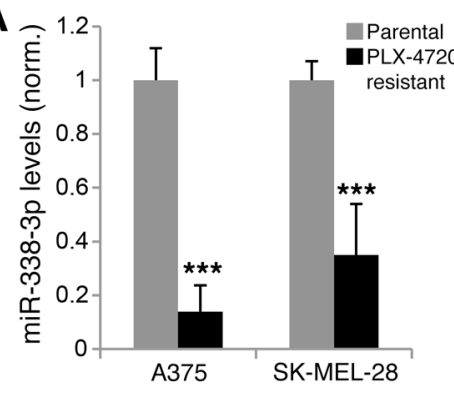

C

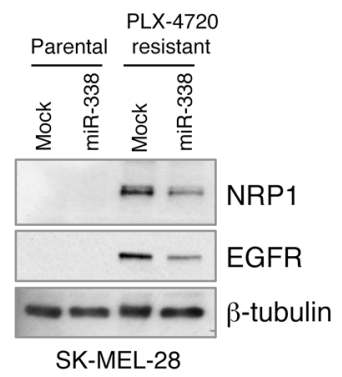

B
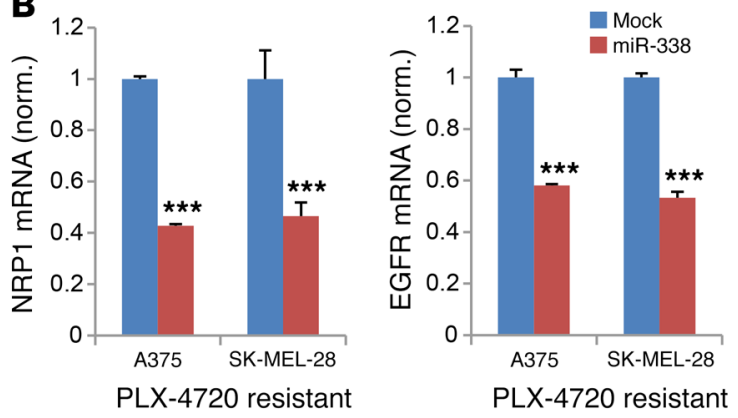

D

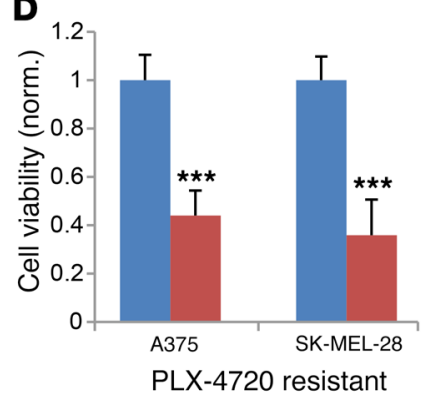

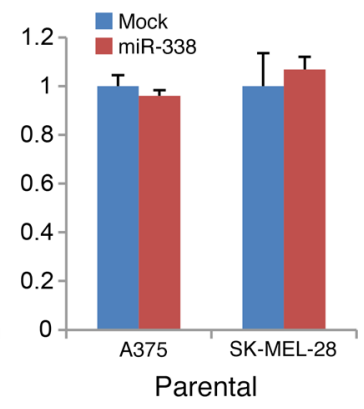

Figure 2. miRNA-338 downregulation mediates NRP1 neoexpression in BRAF inhibitor-resistant melanoma cells. (A) The expression of miRNA-338-3p was assessed by quantitative real-time PCR in PLX-4720-resistant A375 and SK-MEL-28 melanoma cells, and normalized versus the respective parental cells $(n=5)$. Student's $t$ test, ${ }^{* *} P<0.0001$. (B) qPCR analysis of NRP1 (left graph) and EGFR (right graph) expression in drug-resistant A375 and SK-MEL-28 cells, transfected in order to reexpress miR-338 (values normalized to respective mock-transfected cells) $(n=4)$. (C) NRP1 expression assessed by immunoblotting in parental and PLX-4720-resistant A375 cells transfected with pre-miRNA-338 (or mock transfected); vinculin provided a protein loading control (1 representative experiment of 5 repetitions). (D) Viability of drug-resistant (or parental) A375 and SK-MEL-28 cells, transfected with miR-338 or mock (as shown in B; values normalized to respective mock-transfected cells) $(n=3)$.

derived from gastric cancer, and EBC1 and H1993 derived from lung cancer. As previously reported, these cells are crucially dependent on MET tyrosine kinase activity and their viability is severely impaired by treatment with the MET-specific kinase inhibitors JnJ-38877605 (JNJ-605) or PHA-665752, or the clinically approved multikinase inhibitor crizotinib (25). These cells constitutively express NRP1 (Figure 3A), but its knockdown by shRNAs (validated in Supplemental Figure 3A) did not affect their viability or proliferation rate (Figure 3B and Supplemental Figure 3B), indicating that these oncogeneaddicted cells are not dependent on NRP1 expression. Nonetheless, NRP1-depleted cells displayed significantly increased sensitivity to anti-Met agents $\left(\mathrm{IC}_{50}\right.$ was reduced by half, or more), as shown by the impaired cell viability and growth in the presence of MET inhibitors both in short-term (Figure 3, C-H) and long-term experiments (Figure 3, depleted melanoma cells. Interestingly, SOX10, in addition to positively controlling the promoter of neural crest lineage differentiation genes, is also known to induce the expression of several micro-RNAs, including miR-338-3p (23), which was reported to downregulate NRP1 expression in cancer cells $(16,24)$. We verified, in a transcriptome data set of 57 different melanoma cell lines, that the expression of SOX1O and miR-338 are strongly correlated (Spearman's correlation coefficient: $0.539 ; P=2.9 \times 10^{-5}$ ), whereas in contrast they are both inversely correlated with NRP1 (Supplemental Figure 2H). Importantly, miR-338 levels were strikingly decreased in $\mathrm{SOX}^{\mathrm{lo}} / \mathrm{NRP} 1^{\text {hi }}$ drug-resistant $\mathrm{A} 375$ and SK-MEL-28 melanoma cells (Figure 2A). Conversely, miR-338 reexpression in resistant cells strongly reduced NRP1 and EGFR mRNA and protein levels (Figure 2, B and C). Moreover, drug sensitivity was restored in resistant cells upon miR-338 forced reexpression (Figure 2D). These data identify a SOX10-miR338-NRP1 regulatory axis, which could account for the low levels of NRP1 in neural crest-derived cells, including melanocytes and melanoma cells. Moreover, this could explain the increase in NRP1 expression (upon SOX10 and miR-338 downregulation) in BRAF inhibitor-resistant cells.

NRP1 controls response to targeted therapy in MET oncogeneaddicted cancer cells. In order to generalize the above findings and extend the relevance of NRP1 to cancer cells addicted to different oncogenes, we analyzed 3 carcinoma cell lines carrying $M E T$ oncogene amplification and constitutive signaling: namely, GTL16
I and J). Altogether, our data in $B R A F$ and $M E T$ oncogene-addicted cells suggest that NRP1 expression becomes crucial when the driver pathway sustaining proliferation is blocked by targeted inhibitors.

In a complementary approach, since adaptive (de novo) NRP1 expression in melanoma cells is required to drive resistance to BRAF inhibitors, we asked whether enhanced NRP1 expression in MET-addicted cancer cells could impair sensitivity to targeted therapy. Indeed, stably NRP1-overexpressing cells demonstrated relative refractoriness (or overt primary resistance) to targeted inhibitors, with remarkably increased $\mathrm{IC}_{50}$ values compared with controls (Figure 4, A and B). Consistent with the findings in drug-resistant melanoma cells, these data suggest that increased NRP1 levels confer a selective advantage to MET-addicted cancer cells subjected to targeted therapy. In order to test this hypothesis in vivo, we established heterogeneous tumor xenografts in mice by injecting a mix of both MET-addicted carcinoma cells overexpressing NRP1 and those mock transduced. Notably, in each experimental group, 1 of the 2 cell populations had been fluorescently labeled to score its representation in the growing tumors. In the absence of any selective therapeutic pressure, both cancer cell populations showed similar growth rates in vivo (Figure 4C, solid lines). In contrast, upon treatment with a MET inhibitor (dotted lines), control cells were selectively curbed (further validated in Supplemental Figure 4, A and B), enabling the overgrowth of NRP1-overexpressing cells within the heterogeneous tumors (Figure 4C). Indeed, based on the fluorescent signal (Figure 4C) and 
A
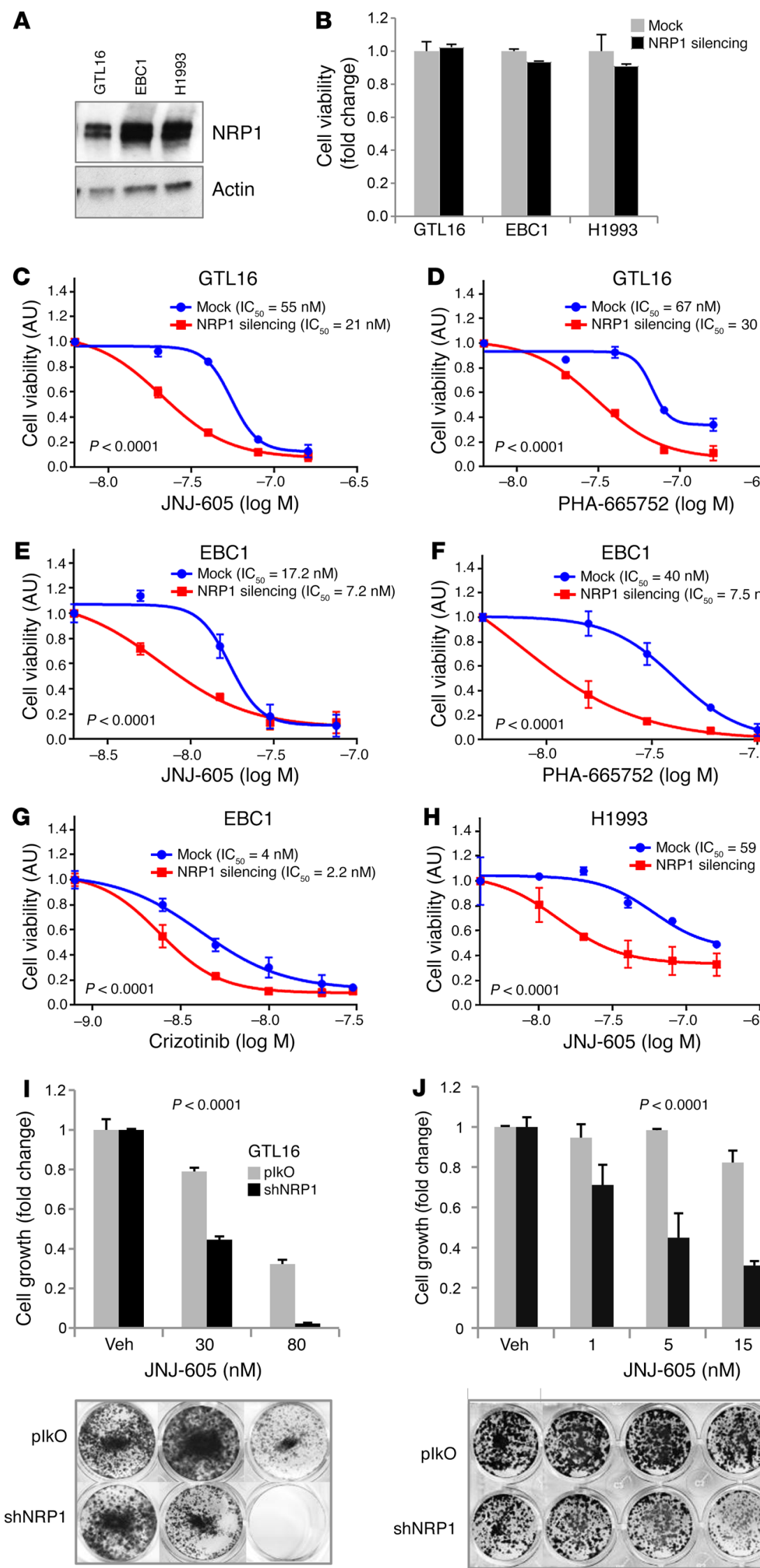
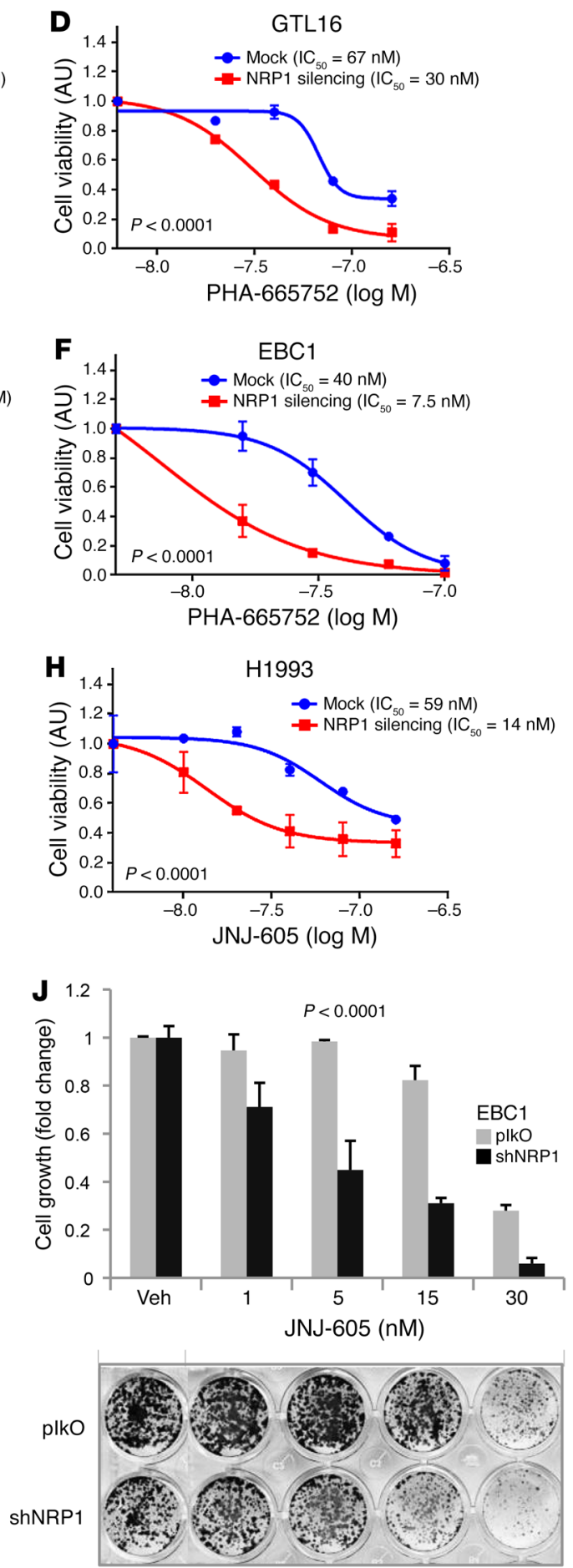

Figure 3. NRP1 controls the therapeutic response to targeted therapy in MET oncogene-addicted cancer cells. (A) NRP1 expression was assessed by immunoblotting in GLT16 (gastric cancer), EBC1 (lung cancer), and H1993 (lung cancer) Metaddicted cells; $\beta$-actin provided a protein loading control (1 representative experiment of 2 repetitions). (B) The viability of the Met-addicted cells described in $\mathbf{A}$ was assessed by Cell Titer Glo Viability Assay upon NRP1 knockdown by RNA interference $(n=3)$. (C-H) The viability of GTL16 (C and D), EBC1 (E-G), and H1993 cells (H), either NRP1-depleted or mock-transfected (same cells as in B), was assessed in the presence of increasing concentrations of the Met inhibitors indicated below the graphs: JnJ-38877605 (JNJ-605), PHA-665752, or crizotinib $(n>5)$. $\mathrm{IC}_{50}$ values were calculated by Graphpad. (I and J) The growth of NRP1depleted or mock-transfected GTL16 (I) and EBC1 (J) (same cells as in previous panels) was assessed by staining with crystal violet and reading Abs $595 \mathrm{~nm}$ after 2 weeks in culture in the presence of the indicated concentrations of the Met inhibitor JNJ-605 and normalized to untreated control cells condition $(n=3)$. In C-J, the statistical analysis was done by 2-way ANOVA with Bonferroni's correction, comparing the behavior of NRP1-silenced and control cells. 

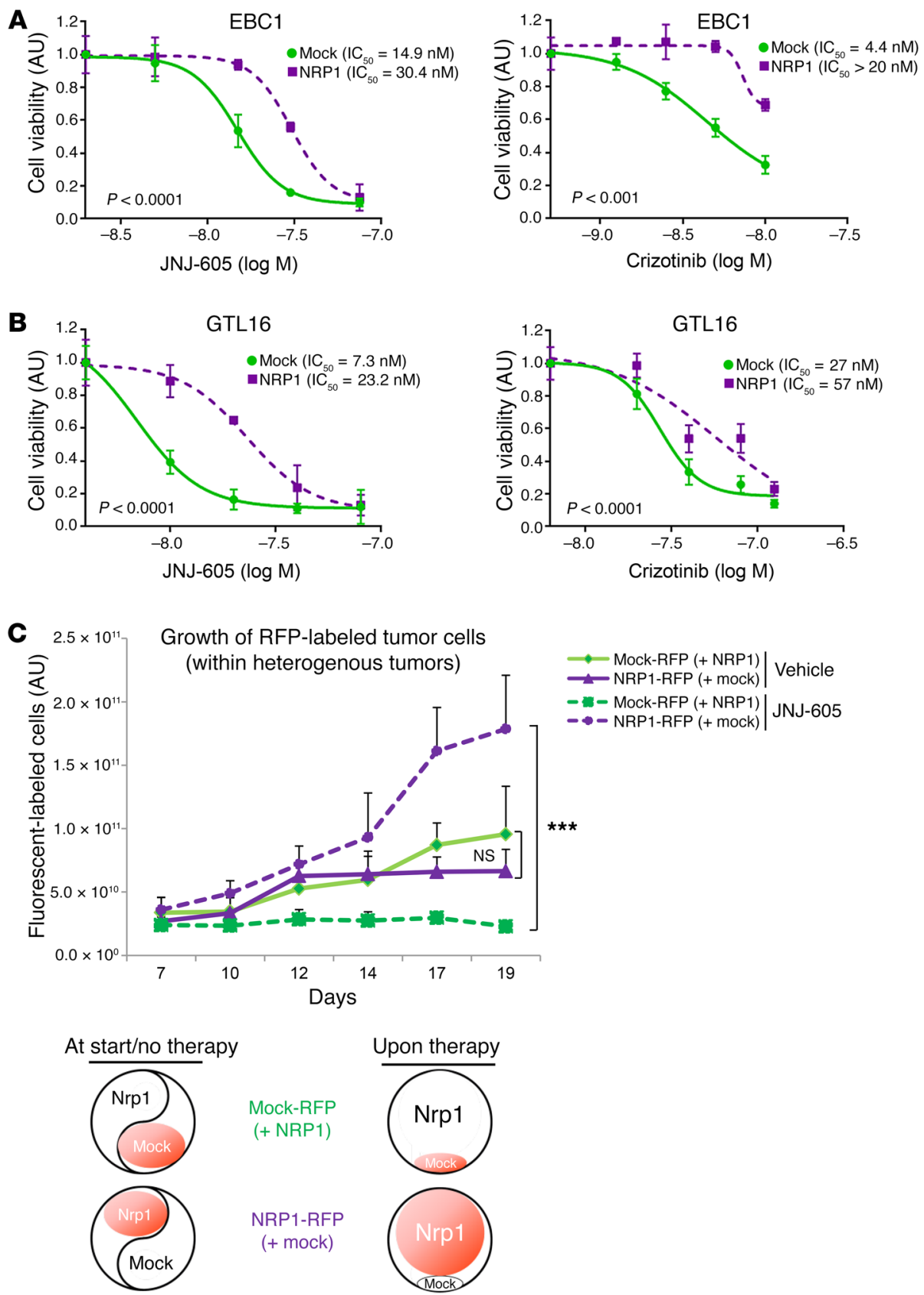

a genetic analysis of explanted tumors (Supplemental Figure 4, $\mathrm{A}$ and $\mathrm{B}$ ), we found that $\mathrm{NRP}^{\mathrm{hi}}$ cells had acquired striking drug refractoriness and selective growth advantage, overwhelming the mock cell population and enabling tumor development even in presence of targeted therapy (Supplemental Figure 4, B-D).

From a mechanistic perspective, we demonstrated that NRP1 overexpression in MET-addicted carcinoma cells upregulated endogenous EGFR levels (Figure 5A and Supplemental Figure $4 \mathrm{E})$. Interestingly, as in melanoma, a strong correlation between NRP1 and EGFR mRNA levels was observed in data sets derived from human lung (TCGA), breast (Metabric), and colorectal (TCGA) cancer samples (Spearman's correlation coefficients: $0.470,0.376$, and 0.252 , respectively; $P<0.00001$ for all). Notably, NRP1 upregulation in MET-addicted cells correlated with increased EGFR tyrosine phosphorylation and persistence of acti-
Figure 4. NRP1 expression confers refractoriness to targeted therapy and selective growth advantage in vivo to Met-addicted cells. (A and $\mathbf{B}$ ) The viability of EBC1 (A) or GTL16 (B) Met-addicted cells, either NRP1 overexpressing or mock transfected, was assessed in the presence of increasing concentrations of the indicated Met inhibitors $(n>6)$. $\mathrm{IC}_{50}$ values were calculated by Graphpad. (C) NOD-SCID mice were injected subcutaneously with a heterogeneous mix of EBC1 cells, half control and half overexpressing $\operatorname{NRP} 1(n=5$, per experimental condition). In 2 complementary settings, one of the cell populations was previously marked by fluorescent Turbo-RFP, featuring a mockRFP-labeled mix and a NRP1-RFP-labeled mix, which allowed us to follow the growth of intermingled fluorescent cells expressing different NRP1 levels by in vivo imaging with the IVIS Spectrum CT system ( $y$ axis). As expected, mouse treatment with the Met-inhibitor JNJ-605 (2.5 mg/kg by daily oral gavage) impaired the growth of control cells (revealed by fluorescent signal in mock-RFP mix tumors in this bar graph, and by gene marker-specific qPCR in Supplemental Figure $4, A$ and $B$ ). However, NRP1-overexpressing cells (revealed by fluorescence in NRP1-RFP mix tumors in this bar graph, and by gene marker-specific qPCR in Supplemental Figure $4 B$ ) were relatively refractory to targeted therapy. Indeed, due to overgrowth of NRP ${ }^{\text {h }}$ cells, all tumors developed irrespective of targeted therapy (see Supplemental Figure 4, C and D). In all panels, the statistical analysis was done by 2-way ANOVA with Bonferroni's correction, comparing the behavior of NRP1 ${ }^{\text {hi }}$ and mock-transfected control cells; ${ }^{* * *} P<0.0001$ vated AKT in MET inhibitor-treated cells (Figure 5B), consistent with the notion that an alternative signaling pathway sustains cell viability and growth. The functional relevance of EGFR signaling in NRP1-induced resistance to MET-targeted therapies was finally validated by finding reduced cell viability upon combined treatment with the MET inhibitor in association with EGFR-specific inhibitors erlotinib or cetuximab (Figure 5C).

NRP1-dependent upregulation of IGF1R kinase mediates acquired resistance to targeted therapy in HER2-addicted breast cancer cells. Approximately $20 \%$ of human breast cancers are characterized by HER2 oncogene amplification and constitutive activation, featuring an addiction that is usually therapeutically targeted with HER2 inhibitors. In advanced disease, however, resistance to HER2-targeted drugs almost invariably ensues (26). Intriguingly, by analyzing RNA-Seq data from a panel of matched breast cancer 
A
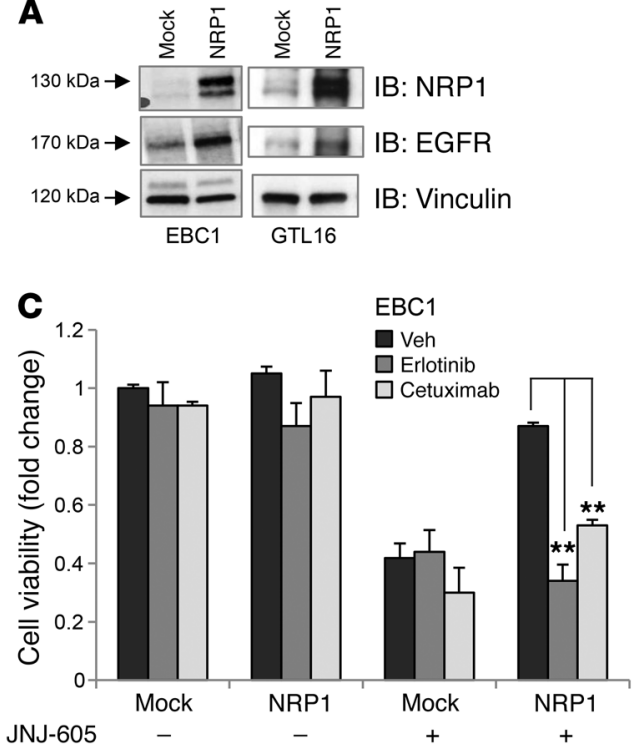

Figure 5. NRP1 upregulates EGFR in carcinoma cells resistant to Met-targeted inhibitors. (A) NRP1 and EGFR expression was analyzed by immunoblotting in the indicated Met-addicted cells, either control or transduced to overexpress NRP1; vinculin provided a protein loading control (1 representative experiment of 5 repetitions). (B) EBC1 cells overexpressing NRP1 (or mock controls) were analyzed by Western blotting to reveal the expression and the phosphorylation levels of Met and EGFR tyrosine kinases, as well as their intracellular effector AKT (1 representative experiment of 4 repetitions; duplicate samples were run on parallel gels). (C) The viability of EBC1 cells overexpressing NRP1 (or mock controls) was assessed in the presence of the Met-inhibitor JNJ-605 (15 nM), either alone or in combination with the EGFR inhibitors cetuximab $(1 \mu \mathrm{g} / \mathrm{ml})$ or erlotinib $(1 \mu \mathrm{M})$. Statistical significance was calculated by Student's $t$ test with Bonferroni's correction, comparing each cetuximab- or erlotinib-treated condition versus respective vehicle-treated conditions $(n=6) ;{ }^{*} P<0.001$.

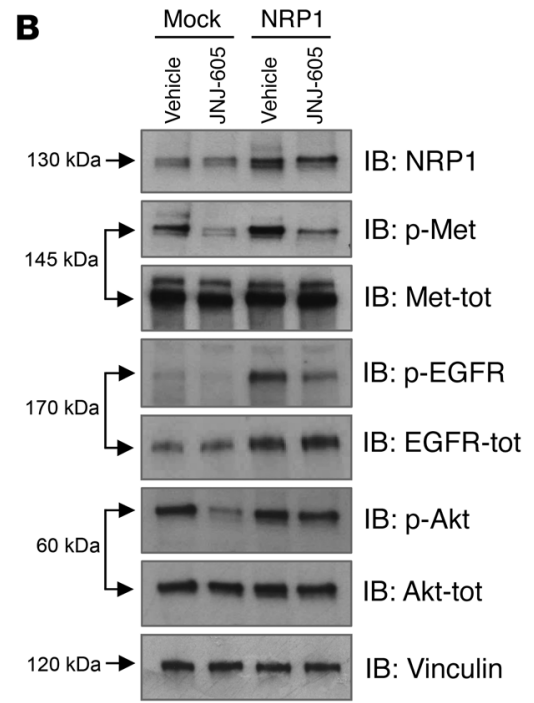

sion in melanoma cells, could play a similar role in breast cancer. Actually, the upstream controller SOX10 is a lineage-specific transcription factor that is not expressed in carcinomas. Consistently, we found that miR-338 was not downregulated in SKBR3 and BT 474 carcinoma cells upon the onset of resistance to HER2 inhibitors (not shown). Thus, NRP1 upregulation associated with resistance to targeted therapy can be due to different mechanisms in different tumor types.

Notably, lapatinib is a dual-kinase inhibitor that, in addition to HER2, targets EGFR. Thus, we were not expecting to find activated EGFR as a resistance mechanism in this experimental model. Indeed, we did not observe HER2 or EGFR phosphorylation in drug-resistant cells, whereas in an unbiased phosphoprotein screening we found a marked upregulation of $I G F 1 R$ expression and activity (Supplemental Figure 6A), which was then validated by Western blotting (Figure 6C). Using the approach followed for investigating BRAF- and MET-addicted cells, we demonstrated the requirement of NRP1 to induce IGF1R in drug-resistant cells (Figure 6D). Moreover, whereas parensamples (and cell lines) before and after targeted therapy (27), in most cases we found NRP1 upregulation in response to treatment with the HER2 inhibitor trastuzumab (Supplemental Figure 5, A and B). We also observed NRP1 upregulation in HER2-addicted BT474 breast cancer cells treated with the targeted inhibitor lapatinib (Supplemental Figure 5C), suggesting an adaptive druginduced response. Notably, the analysis of RNA-Seq data derived from BT474 cells subjected to HER2 silencing clearly indicated increased NRP1 levels (Supplemental Figure 5D), suggesting that NRP1 expression in these cells is basally constrained by constitutively active HER2 signaling.

We therefore set out to test the role of NRP1 in the responsiveness of HER2-addicted cancer cells to targeted therapies, by treating BT474 and SKBR3 with escalating doses of lapatinib until the onset of drug resistance. Indeed, we found strong upregulation of NRP1 in drug-resistant cells, both at mRNA and protein levels (Figure 6A and Supplemental Figure 5E). Moreover, gene silencing confirmed the functional requirement of NRP1 in mediating resistance to targeted therapy (Figure 6B), whereas its depletion had no significant impact on the viability of parental HER2-addicted cells. Using a complementary approach, we found that NRP1 overexpression was sufficient to confer resistance to lapatinib treatment in the same cells (Supplemental Figure 5F). These data confirmed the concept that NRP1 upregulation is a mechanism that sustains cancer viability and proliferation in a range of cancer cells. We also asked whether miR-338, which we found to regulate NRP1 expres- tal breast cancer cells were not dependent on IGF1R, their drugresistant derivatives relied on IGF1R activity to sustain refractoriness to HER2-targeted therapy (Supplemental Figure 6B). Intriguingly, we verified that IGF1R was not induced in melanoma cells upon the onset of BRAF-inhibitor resistance (Supplemental Figure 6C), nor was it implicated in the loss of drug efficacy (Supplemental Figure 6D), indicating that NRP1 can sustain diverse mechanisms of refractoriness to therapy mediated by distinctive RTKs.

NRP1 drives EGFR and IGF1R upregulation via JNK kinase activity and SOX2/JUN transcription factors. We set out to investigate the molecular mechanisms by which the NRP1-induced signaling cascade elicits EGFR and IGF1R transcriptional upregulation in drug-refractory cells. Intriguingly, it was reported previously that NRP1, via its intracellular adapter GIPC, negatively regulates the CDK-inhibitor p27-Kip1 $(10,28)$, which in turn is known to curtail EGFR levels by inhibiting the upstream regulator JNK (29). Indeed, JNK kinase is one of the intracellular signal transducers activated by NRP1 (30). We found p27 downregulation at mRNA and protein levels (Figure 7, A and B), and increased phosphorylated (active) JNK in drug-resistant melanoma and breast cancer cells (compared with parental), as well as in NRP1-overexpressing EBC1 cells (Figure 7B). Conversely, NRP1 downregulation caused by miR-338 reexpression in drug-resistant melanoma cells triggered a cascade leading to p27 increase and pJNK suppression (Supplemental Figure 7A). In keeping with these data, silencing endogenous NRP1 levels in EBC1 carcinoma cells overtly 
A
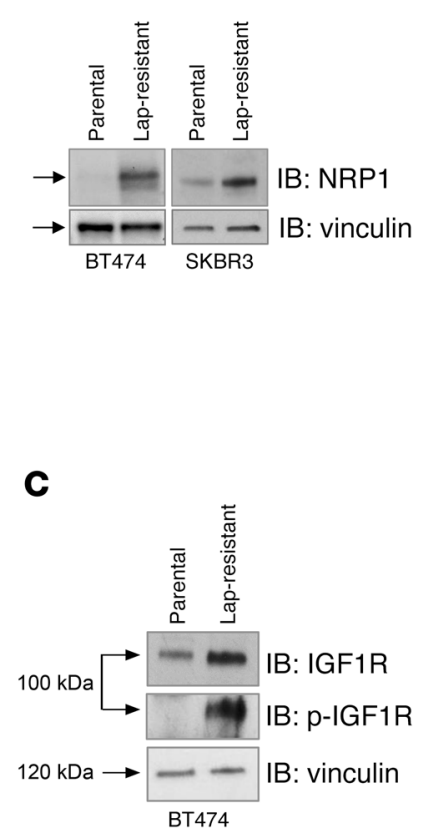

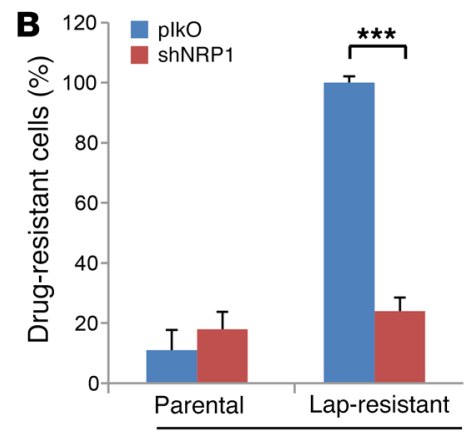

BT474
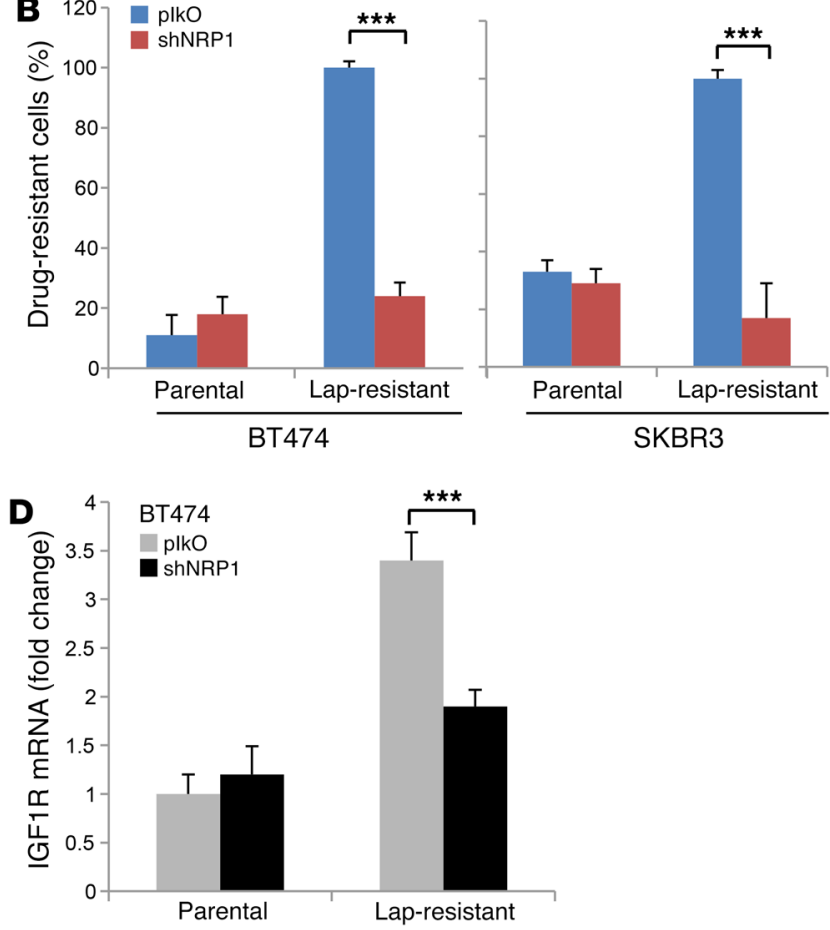

Figure 6. NRP1 upregulation in HER2-addicted breast carcinoma cells mediates acquired resistance to targeted therapy. (A) Immunoblotting analysis of NRP1 expression in the indicated breast cancer cells, either parental naive or with acquired resistance to the HER2-inhibitor lapatinib (Lap) (250 nM for BT474 and 120 nM for SKBR3); vinculin provided a protein loading control (1 representative experiment of at least 3 repetitions). (B) The same cells described in A were subjected to NRP1 knockdown by shRNAs (or treated with plkO empty vector); the fraction of cells resistant to lapatinib (same concentrations as indicated above) was defined as residual viability in presence of the drug versus untreated conditions $(n>4)$. (C) Immunoblotting analysis of IGF1R expression and tyrosine phosphorylation in lapatinib-resistant BT474 cells; vinculin provided a protein loading control (1 representative experiment of 3 repetitions). (D) IGF1R mRNA expression levels were measured by qPCR in parental or lapatinib-resistant BT474 cells, either control or upon NRP1 silencing $(n=3)$. Statistical significance was assessed by Student's $t$ test; ${ }^{* * *} P<0.0001$.

upregulated p27 and repressed pJNK (Figure 7C). Moreover, the mechanistic role of the NRP1-associated adapter protein GIPC in this pathway was validated by knockdown experiments in NRP1dependent drug-resistant cells, resulting in increased p27 and consequent impairment of EGFR or IGF1R upregulation (Supplemental Figure 7B). Notably, p27 reexpression by transfection was sufficient to revert NRP1-dependent pJNK induction and EGFR upregulation (Figure 7D and Supplemental Figure 7, C and D). Importantly, inhibition experiments showed that JNK kinase activity is required to mediate this novel NRP1-induced signaling cascade leading to EGFR or IGF1R upregulation in diverse drug-refractory cells (Figure 7, E-G and Supplemental Figure 7E).

Hence, we asked about transcription factors regulated by JNK and potentially implicated in the upregulation of EGFR and IGF1R. One candidate was SOX2, an upstream regulator of EGFR expression $(31,32)$, previously found to be associated with cancer cell stemness and resistance to targeted therapy (33). Notably, we discovered that SOX2 levels were dramatically increased in BRAF inhibitor-resistant melanoma and NRP1-overexpressing carcinoma cells, in a JNK kinase-dependent manner (Figure 8A). In fact, SOX2 silencing reverted NRP1-dependent induction of EGFR in drugresistant cells (Figure 8, B and C and Supplemental Figure 7F). inhibition per se did not have a significant impact on cancer cell viability; however, drug-refractory melanoma and breast cancer cells were significantly resensitized to oncogene-targeted drugs when cotreated with a JNK inhibitor (Supplemental Figure 7I). Moreover, the presence of JNK inhibitor prevented the development of drug resistance that usually arises in SK-MEL-28 melanoma cells after 5 weeks of treatment with the BRAF inhibitor PLX-4720 (Figure 8G).

NRP1-interfering molecules extend the efficacy of targeted therapy and resensitize drug-resistant cells to oncogene inhibitors. In order to validate the potential translational relevance of targeting NRP1 protein to improve the therapeutic response to oncogene-targeted drugs, we assayed combined kinase inhibitor treatments with NRP1-interfering molecules. Specifically, we applied the small molecule NRP1 inhibitor EG00229, previously found to interact with the extracellular b1b2 domain of NRP1 and to prevent the transactivation of the neuropilin-associated tyrosine kinase VEGFR2 (36). In addition, we generated NRP1-specific nanobodies, which are camelid single-domain antibody fragments (37). The generation and characterization of these nanobodies are described in detail in Methods. Of note, nanobody HS45 (Nb-HS45) recognizes human NRP1 with a high affinity $\left(\mathrm{K}_{\mathrm{D}}: 4.23\right.$ $\mathrm{nM}$ ), whereas it does not recognize its orthologue NRP2. Treat- 

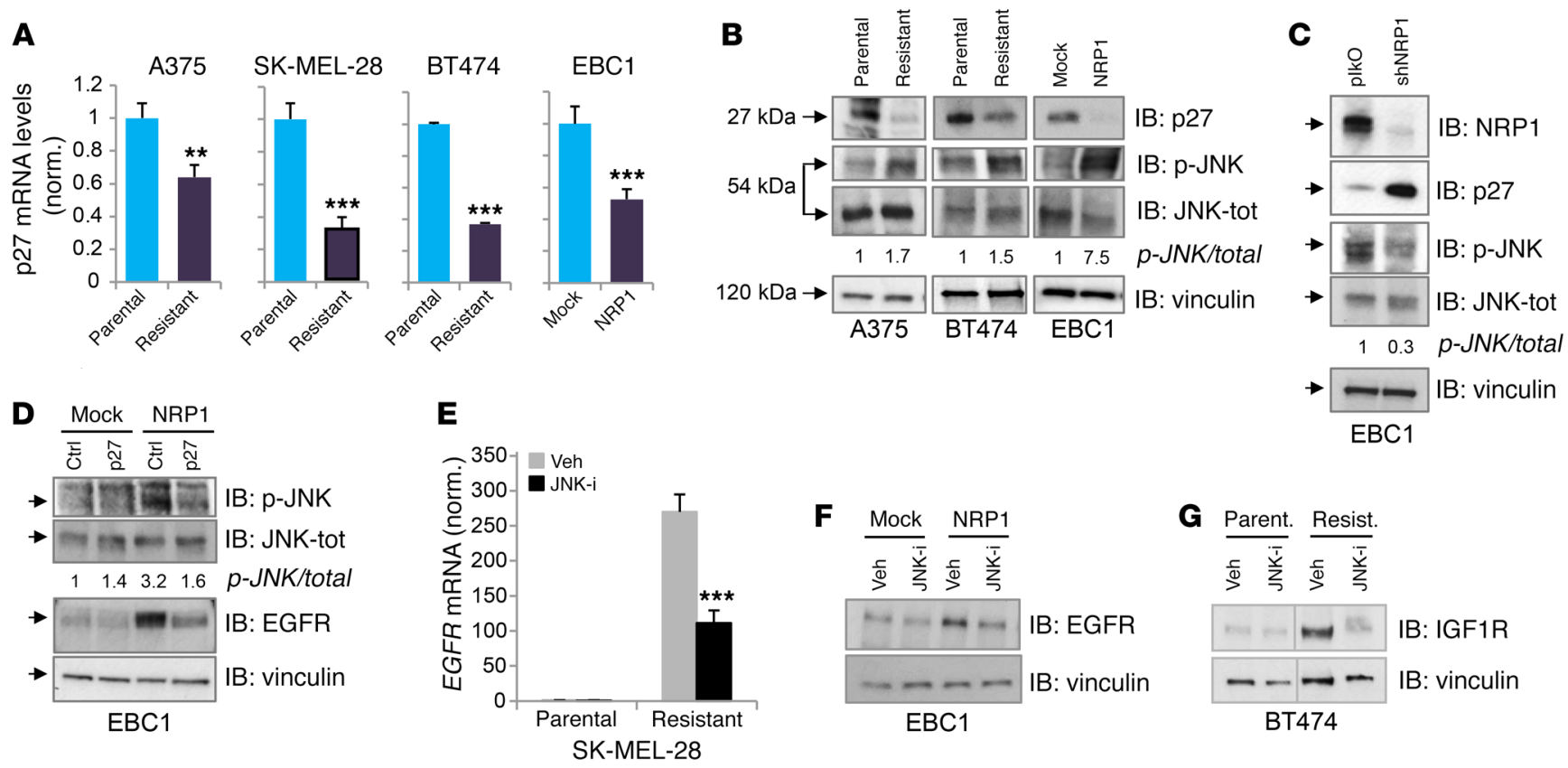

Figure 7. p27 decrease and JNK activation mediate NRP1-driven EGFR and IGF1R upregulation. (A) p27 mRNA levels were measured by qPCR ( $n>3$ ) in the indicated cancer cell lines: A375 and SK-MEL-28 (parental or resistant to PLX-4720), BT474 (parental or resistant to lapatinib), and EBC1 (NRP1 overexpressing or mock transfected). (B) p27-Kip1, activated phospho-JNK, and total JNK protein levels in cancer cells described in A (1 representative experiment of 4 repetitions; duplicate samples were run on parallel gels). The values at the bottom indicate densitometric measurement of relative $p$-JNK versus total JNK band intensity. (C) NRP1, p27, p-JNK, and total JNK levels in EBC1 cells subjected to NRP1 silencing (1 representative experiment of 3 repetitions; duplicate samples were run on parallel gels). (D) p-JNK and EGFR levels in EBC1 cells (shown in B), subjected to p27 overexpression (1 representative experiment of 3 repetitions; duplicate samples were run on parallel gels). (E) qPCR analysis of EGFR expression in SK-MEL-28 melanoma cells, parental or PLX-4720 resistant, upon treatment with the JNK kinase inhibitor SP600125 (25 $\mu \mathrm{M}$; JNK-i) or with vehicle alone $(n=5)$. (F) EGFR expression in EBC1 cells (shown in B), either in basal conditions (Veh) or in the presence of $25 \mu \mathrm{M}$ SP600125 (1 representative experiment of 6 repetitions). (G) IGF1R levels in BT474 cells (shown in B), either in basal conditions or in the presence of $25 \mu \mathrm{M}$ SP600125 (1 representative experiment of 5 repetitions). ${ }^{* *} P<0.001,{ }^{* * *} P<0.0001$.

ment with either of these NRP1-interfering molecules enhanced the activity of MET kinase inhibitors and promoted cancer cell viability loss at significantly lower drug concentrations, with up to 8-fold-reduced $\mathrm{IC}_{50}$ (Figure 9A and Supplemental Figure 8, A-C). Notably, upon MET signaling blockade, ligand-dependent EGFR activation could sustain intracellular AKT and MAPK signaling in these cells, whereas NRP1-blocking nanobodies negated this effect (Supplemental Figure 8D), indicating inability of the EGFR pathway to mediate survival after targeted therapy. Similarly, we found that combined treatment with EG00229 restored the therapeutic response to lapatinib in drug-resistant breast cancer cells (Figure 9B). We finally tackled the NRP1-dependent onset of resistance to BRAF inhibitors in SOX10-deficient melanoma cells, by applying EG00229 or anti-NRP1 nanobodies, and found that this could prevent EGFR upregulation (Figure 9C) and acquired refractoriness to targeted therapy (Figure 9D). Furthermore, the functional inhibition of NRP1 in PLX-4720-resistant melanoma cells resulted in a significant recovery of drug sensitivity and longterm therapeutic response (Figure 9E). Notably, EG00229 synergized with cetuximab to achieve resensitization of melanoma cells to targeted therapy (Figure 9F).

Thus, NRP1 functional interference strongly enhanced the efficacy of oncogene-targeted therapies and prevented the onset of drug resistance. In consideration of the potential therapeutic relevance of this approach, we wondered whether NRP1-targeted molecules could also affect normal non-oncogene-addicted cells. Actually, neither viability nor growth of primary HUVECs, immortalized normal HEK-293 kidney cells, or 3T3 fibroblasts were affected by treatment with high doses of EG00229 or HS45 nanobodies (Supplemental Figure 9, A-C). Experiments were aimed at revealing potential short- or long-term toxicities. Moreover, we assayed combined treatments with oncogene- and NRP1-targeted molecules, as was done for cancer cells (Supplemental Figure 9D).

\section{Discussion}

A major challenge of precision oncology is dealing with acquired resistance to targeted therapies. Understanding the molecular mechanisms of resistance is therefore of paramount importance to improve treatment efficacy. For instance, melanoma resistance to BRAF inhibitors is accounted for by a range of different mechanisms. In around one-third of cases, the mechanism is epigenetic regulation of gene expression, which often leads to increased receptor tyrosine kinase signaling $(38,39)$. We and others have previously shown that BRAF-addicted melanoma cells, upon treatment with targeted inhibitors, undergo adaptive gene expression reprogramming and develop secondary drug resistance associated with the upregulation of EGFR tyrosine kinase $(17,40)$. Here we show that de novo NRP1 expression in melanoma cells is a pivotal driver of this process. The same drug-resistance mechanism was confirmed in oncogene-addicted carcinoma cells upon 

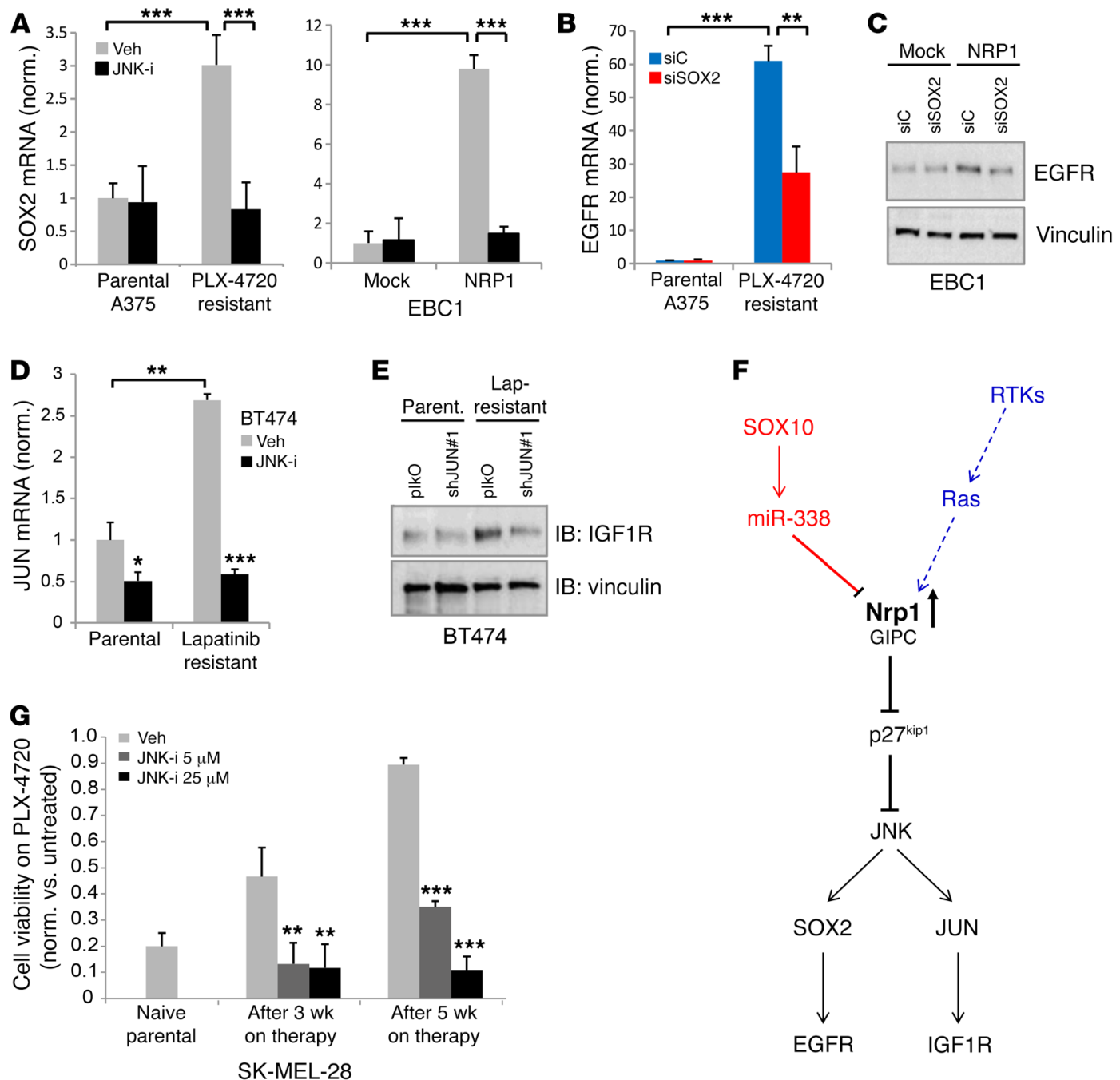

Figure 8. SOX2 and JUN transcription factors mediate NRP1/JNK-driven upregulation of EGFR and IGF1R, respectively. (A) qPCR analysis of SOX2 expression in parental and drug-resistant A375 and EBC1 cells (shown in B), either in basal conditions or in the presence of $25 \mu \mathrm{M}$ SP600125; values normalized to untreated controls $(n=5)$. (B) qPCR analysis of EGFR expression in parental and drug-resistant A375 cells subjected to SOX2 silencing by targeted siRNAs (validated in Supplemental Figure 7F) ( $n=4)$. (C) EGFR expression in EBC1 cells (shown in B), subjected to SOX2 silencing (validated in Supplemental Figure 7F) (1 representative experiment of 4 repetitions). (D) qPCR analysis of JUN expression in parental and drug-resistant BT474 cells, either in basal conditions or in the presence of $25 \mu \mathrm{M}$ SP600125; values normalized to untreated parental cells $(n=3)$. (E) IGF1R expression in BT474 cells (same as above), subjected to JUN silencing by RNAi (validated in Supplemental Figure 7G) (1 representative experiment of 4 repetitions). (F) Schematic working model of the proposed signaling cascade leading to the onset of resistance to oncogene-targeted therapy in different cancer cells. The upstream regulation of NRP1 levels by miR-338-3p (marked in red) was specifically observed in melanoma cells, whereas in carcinoma cells NRP1 expression might depend on RTK-Ras

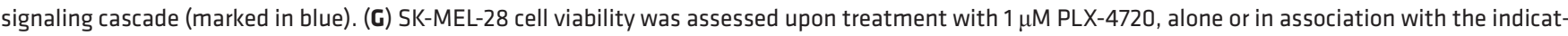
ed concentrations of JNK-i SP600125. While naive cells were initially sensitive to PLX-4720, after 3-5 weeks in culture they progressively developed drug resistance, but this was prevented in the presence of JNK-i $(n>3)$. In all panels, statistical significance was assessed by Student's $t$ test with Bonferroni's correction; ${ }^{*} P<0.01,{ }^{* *} P<0.001,{ }^{* * *} P<0.0001$.

NRP1 overexpression, consistent with the idea that NRP1 acts as a common trigger to upregulate pathways sustaining cancer cell survival and growth under life-threatening conditions. In fact, we and others have previously shown that a range of carcinoma cell lines are dependent on NRP1 for viability and proliferation, which may relate to the ability of NRP1 to stimulate multiple growth factor receptors $(9,12)$. Here we show that cancer cells carrying a constitutively activated oncogene are not dependent on NRP1; however, NRP1 regains its pivotal growth-promoting role when the oncogenic addiction pathway is blocked by targeted inhibitors. Moreover, whereas NRP1 is barely detectable in melanoma cells due to a SOX10/miR-338 pathway targeting its transcript in neural crest-derived cells, this restriction mechanism is blunted in response to target therapy, enabling NRP1 upregulation and the onset of drug resistance. In carcinoma cells, which do not carry the SOX10/miR-338 regulatory axis, the regulation of this miRNA doesn't seem to be associated with drug resistance (not shown); in fact, other mechanisms have been implicated in NRP1 upregulation in carcinoma cells (41-43), and could be postulated to elicit the adaptive response to targeted therapy. Moreover, we report here that NRP1 expression may be unleashed upon inhibition of HER2 constitutive activity in breast cancer cells. 
A

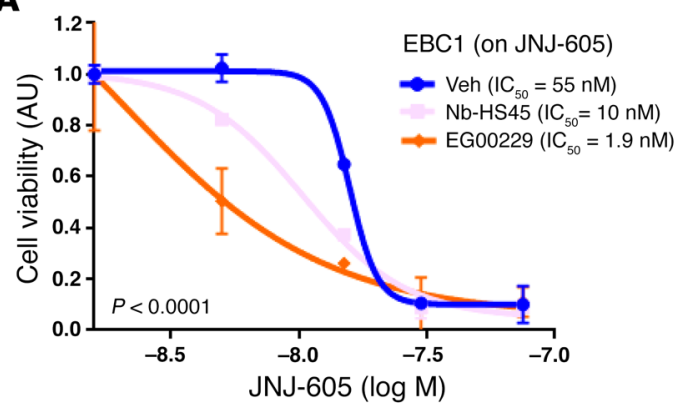

B

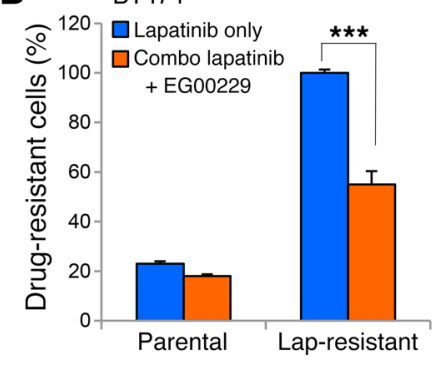

C
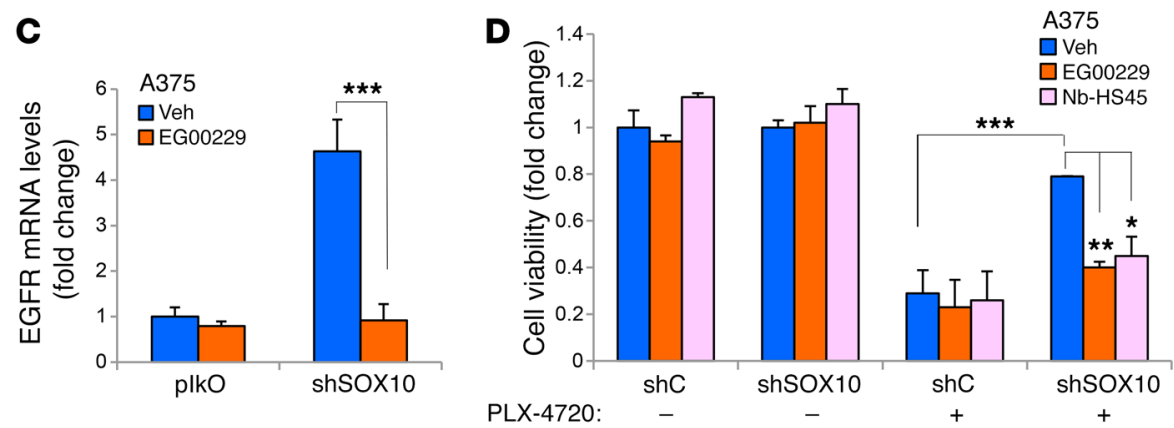

E

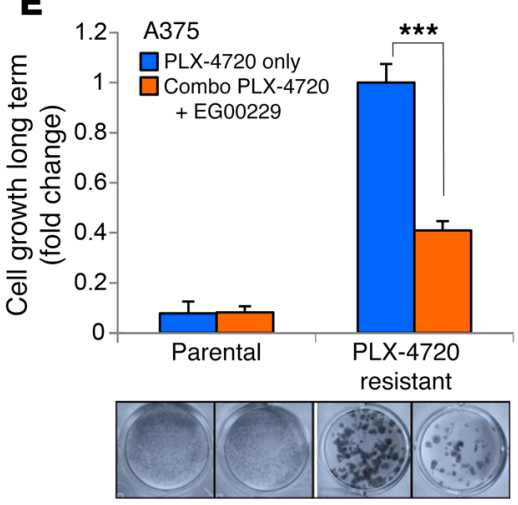

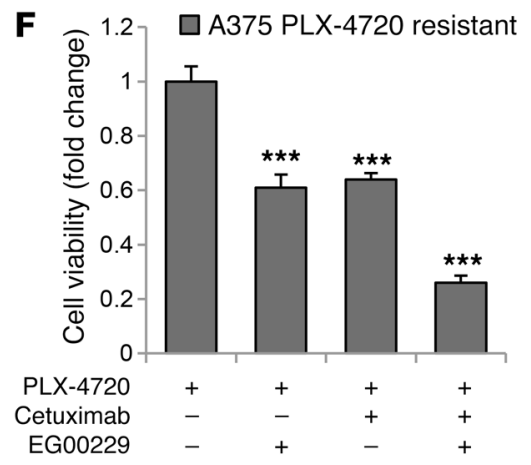

Figure 9. Therapeutic efficacy of NRP1-interfering molecules in combination with BRAF-, HER2-, or Met-targeted inhibitors. (A) The viability of EBC1 Met-addicted cells $(n=5)$ was assessed upon treatment with increasing concentrations of the Met inhibitor JNJ-605, either alone or in combination with the NRP1-targeted molecule EG00229 $(12.5 \mu \mathrm{M})$ or the nanobody HS45 $(5 \mu \mathrm{g} / \mathrm{ml})$. (B) HER2-addicted BT474 breast cancer cells, either parental naive or resistant to the targeted inhibitor lapatinib $(250 \mathrm{nM})$, were exposed to lapatinib alone or in combination with EG00229 $(12.5 \mu \mathrm{M})$; the fraction of drug-resistant cells was calculated based on residual viability versus untreated conditions $(n=4)$. (c) EGFR mRNA levels were measured in S0X10-depleted A375 melanoma cells (or controls) $(n=4)$, treated with the NRP1-targeted molecule EC00229 $(12.5 \mu \mathrm{M})$ or with vehicle alone. (D) Viability of SOX10depleted A375 melanoma cells (or controls) ( $n$ >4) exposed to $0.25 \mu \mathrm{M}$ PLX-4720, either alone or in combination with the small molecule EC00229 $(12.5 \mu \mathrm{M})$ or HS45 nanobody $(5 \mu \mathrm{g} /$ $\mathrm{ml})$. (E) The growth of A375 melanoma cells, parental or PLX-4720 resistant $(n=3)$, was assessed after 2 weeks of culture in the presence of the BRAF inhibitor PLX-4720, either alone or in combination with $12.5 \mu \mathrm{M}$ EG00229 (representative images at the bottom). (F) Residual viability of BRAF inhibitor-resistant A375 melanoma cells subjected to different therapeutic associations of PLX-4720 $(2 \mu \mathrm{M})$ with cetuximab $(1 \mu \mathrm{g} / \mathrm{ml})$ and/or EG00229 (12.5 $\mu \mathrm{M})$; the statistical analysis compared combinatorial treatments with PLX-4720 alone ( $n=$ 5). We applied ANOVA tests with Bonferroni's correction to analyze multiple sample comparisons in $\mathbf{A}, \mathbf{D}, \mathbf{F}$; in the other cases, we used Bonferroni-corrected Student's $t$ tests; ${ }^{*} P<0.01,{ }^{*} P<0.001,{ }^{*}{ }^{*} P<0.0001$.
We have previously shown that NRP1 promotes ligandinduced EGFR receptor clustering at the cell surface (9). However, here we demonstrate that NRP1 is also an important trigger of EGFR upregulation at a transcriptional level, which further empowers its activity in support of cancer cell viability and growth. In keeping with these findings, bioinformatic analysis of human tumor sample data sets demonstrated a strong direct correlation between NRP1 and EGFR gene expression in melanomas, as well as lung, colorectal, and breast carcinomas. We found that NRP1-dependent EGFR upregulation is elicited through a novel signaling cascade mediated by JNK kinase and the transcription factor SOX2. Notably, both NRP1 and SOX2 have been previously associated with cancer cell stemness $(21,44)$; thus, future studies may address the question of whether the functional role of NRP1 in stem cell renewal could be mediated by SOX2 activity. Our data unveil a novel function of JNK kinase, eliciting SOX2 and EGFR upregulation in cancer cells. Our findings do not exclude that other factors besides NRP1 could control EGFR levels in cancer. For example, TGFß1 can also induce EGFR expression in melanoma cells (17), and intriguingly, NRP1 has been reported to enhance
TGF $\beta 1$ signaling as well as other potentially relevant growth factor pathways $(11,12)$. In fact, here we demonstrate that NRP1 is critically required to mediate and maintain adaptive EGFR upregulation in melanoma cells and resistance to BRAF-targeted therapies, both in vitro and in mouse models. Moreover, a combined treatment with a NRP1 inhibitor and cetuximab, targeting both EGFR expression and signaling, could achieve full resensitization to therapy of drug-resistant melanoma cells.

Interestingly, NRP1 has been implicated in the control of additional tyrosine kinase receptors besides EGFR, putatively accounting for its ability to promote the growth of a wide range of cancer cells. In this line, we found that NRP1 is also upregulated in HER2-addicted breast cancer cells subjected to targeted therapy. NRP1 is pivotal in this system to induce IGF1R tyrosine kinase expression and activity, a mechanism known to mediate resistance to HER2-targeted inhibitors (45). Similar to what we observed for melanoma, NRP1 upregulation is frequently seen in breast cancer samples upon treatment with HER2 inhibitors. Prospective studies will be required to assess if NRP1 upregulation is a common trait in HER2-amplified breast cancers developing resis- 
tance to targeted therapy. In the present work, we demonstrated that NRP1-dependent IGF1R upregulation in breast cancer cells also requires JNK kinase, whereas c-JUN, a different transcription factor instead of SOX2, is implicated downstream. Altogether, these findings underscore a broad relevance of NRP1 activity in the onset of multiple adaptive mechanisms of cancer cell resistance to therapies targeting oncogene addiction.

From a mechanistic perspective, we found that NRP1-dependent upregulation of both EGFR and IGF1R is elicited through a novel signaling cascade conserved in diverse cancer cells, and is mediated by JNK kinase, which gets activated upon NRP1-dependent downregulation of the inhibitor $\mathrm{p} 27$, consistent with previous data $(10,28)$. This novel pathway seems to rely on the function of the adapter protein GIPC/synectin, which is known to interact with the NRP1 intracellular domain (46). However, the implicated mechanisms leading to NRP1-dependent p27 downregulation need to be clarified. Furthermore, although increased NRP1 protein expression is sufficient to trigger this pathway in cancer cells, it is presently unknown whether the mechanism is ligandindependent or not. Intriguingly, our data suggest that VEGF signals are not implicated. Notably, the pivotal role of JNK in this pathway was further confirmed by the association of a JNK inhibitor with therapies targeting oncogenic addiction, an approach that could effectively prevent the onset of drug refractoriness and partly revert it in stably drug-resistant cells.

Considering the impact of NRP1 signaling on multiple signaling pathways bolstering cancer cell viability and growth, this adaptive surge of NRP1 in response to therapy is posited to be an important mechanism of acquired drug resistance in cancer patients, consistent with what we showed in vitro and in preclinical mouse models. Moreover, NRP1 levels strongly affected up front the sensitivity to targeted drugs of carcinoma cells addicted to the Met oncogene, consistent with the idea that elevated expression of NRP1 in diverse tumors is mechanistically associated with poor response to oncogene-targeted therapies. In a translational perspective, NRP1interfering molecules with potential clinical application in combined therapeutic regimens could include targeted nanobodies or small molecules interacting with the extracellular domain, such as those we found capable of preventing or delaying the onset of resistance in melanoma cells, or even of reverting established drug resistance in experimental models. Interestingly, our data suggest that these NRP1-targeting molecules do not cause nonspecific cell toxicity in culture. However, in vivo experiments are warranted to support further developments in therapeutic perspective. Notably, it can be envisaged that HS45 nanobodies act in a different manner compared with classical anti-NRP1 antibodies previously tested in cancer therapy, which were selected for interfering with VEGFinduced functions. Indeed, NRP1-dependent resistance to target therapies doesn't seem to be associated with VEGF signaling.

Although in the present study we focused on the role of NRP1 in cancer cells, this molecule is also widely expressed in the microenvironment (e.g., in endothelial cells of tumor vessels) as well as in tumor-associated macrophages (TAMs) and regulatory T lymphocytes (Tregs) (12). In these cell types, NRP1 was also reported to promote tumor development, and its cell-specific genetic depletion in mice led to defective TAM recruitment, reduced tumor vasculature and tumor growth, and increased antitumor immune response
$(47,48)$. These data suggest that therapeutic tools interfering with NRP1, applied in combination with oncogene-targeted therapies, could be used not only to prevent drug-resistance in cancer cells, but also to promote environmental conditions favorable to cancer cell clearance. Future studies validating specific NRP1-interfering molecules applicable in vivo for this purpose should therefore be performed in immunocompetent murine preclinical models.

In sum, our study provides the rationale for targeting NRP1dependent mechanisms responsible for adaptive resistance to the blockade of activated oncogenes.

\section{Methods}

Human cell lines. HEK-293T kidney cells, A549 and H1993 lung adenocarcinoma cell lines, and the A375 melanoma cell line were provided by American Type Culture Collection (ATCC). SK-MEL-5, SK-MEL-28, and SK-MEL-2 melanoma cells were provided by the National Cancer Institute (NCI). The BT474 breast ductal carcinoma cell line was provided by DMSZ, and SKBR3 breast adenocarcinoma was provided by the Interlab Cell Line Connection (ICLC). EBC1 human lung squamous cell carcinoma line was purchased from the Health Science Research Resources Bank (HSRRB). GTL16 is a clonal cell line established in our laboratory and derived from the poorly differentiated gastric carcinoma cell line MKN45. Primary HUVECs were cultured in EGM2 medium (Lonza). All tested and authenticated cancer cell lines used in this study were cultured in the following media: RPMI for EBC1, GTL16, H1993, A549, BT474, SK-MEL-5, SK-MEL-28, and SK-MEL-2 cells; and DMEM for HEK-293T, SKBR3, and A375 cells. The media were supplemented with $1 \%$ L-glutamine (2 mM), 10\% FBS (Sigma), penicillin (5,000 U/ml, Faber), and 0.1\% streptomycin ( $5 \mathrm{mg} / \mathrm{ml}$, Squibb) and incubated in a humidified incubator with $5 \% \mathrm{CO}_{2}$ at $37^{\circ} \mathrm{C}$. Cell lines were passaged in culture for fewer than 6 months after resuscitation.

Analysis of mRNA expression in tumor samples. We compared SOX1O, $N R P 1$, and EGFR mRNA levels in 12 matched melanoma samples, each derived from the same patient before and while undergoing treatment with BRAF inhibitors. Ten of these were described in the study published by Kwong and coworkers (49) (data set accession: EGAD00001001306 at www.ebi.ac.uk), whereas an additional 2 were described in our previous study (17). We compared NRP1 mRNA levels in 11 matched breast cancer samples, each derived from the same patient before and while undergoing treatment with HER2 inhibitor (27). Raw data files can be found in NCBI's Gene Expression Omnibus database (GEO GSE60182).

Antibodies and other reagents. Anti-neuropilin-1 antibody used for Western blot analysis was purchased from Abcam (ab813121). EGFR was detected by antibodies purchased from Santa Cruz Biotechnology (clone 1005; sc-03) or Enzo Life Sciences (ALX-804-064-C100). EGFR phosphorylation was detected by a phospho-specific antibody from Abcam (directed to $\mathrm{p}-\mathrm{Tyr}_{1068}$; catalog ab5644). SOX10 antibody was purchased by Abcam (ab155279). Anti-IGF1R $\beta$ was purchased by Santa Cruz Biotechnology (sc-713). GIPC antibody was provided by Santa Cruz Biotechnology (sc-35475). Total and phosphorylated MAPK and AKT (pAKT-S ${ }^{473}$ and pMAPK-Thr ${ }^{202} / \mathrm{Tyr}^{204}$ ) and pJNK (catalog 4668) were detected with antibodies from Cell Signaling. Other antibodies applied in this study were anti-vinculin (V4505, Sigma), anti- $\beta$-actin (clone I-19, Santa Cruz Biotechnology), and anti- $\beta$-tubulin (T4026, Sigma). Secondary antibodies were purchased from Promega or Jackson Laboratories. Phospho-RTK Array (catalog ARY001B) was purchased from R\&D Systems. PLX-4720 was purchased from Med- 
ChemExpress. JNJ-38877605 (JNJ-605) was from Selleck Chemicals. JNK inhibitor SP600125 was provided by Sigma-Aldrich. IGF1R inhibitor BMS-754807 was purchased from Sequoia Research Products. The above inhibitors were resuspended in DMSO as vehicle.

Nanobody generation. Anti-NRP1 single-domain antibody (also known as nanobody [Nb]) HS45 was isolated from an immune $\mathrm{Nb}$ phage-display library as previously described (37, 50). An alpaca (Vicugna pacos) was immunized via an alternating immunization schedule: an injection of $100 \mu \mathrm{g}$ recombinant human NRP1 (R\&D Systems) was followed by $100 \mu \mathrm{g}$ recombinant mouse NRP1 protein (R\&D Systems) 1 week later. This alternating schedule was maintained for a total of 6 weeks; the final injection contained both human and mouse Nrp1. Both proteins were mixed with Gerbu LQ3000 adjuvant before injection. Peripheral blood lymphocyte mRNA was converted into cDNA, from which $\mathrm{Nb}$-coding sequences were amplified and ligated onto the pHEN4 phagemid vector (50). Using M13KO7 helper phages, the Nb library was expressed on bacteriophages in E. coli, and specific $\mathrm{Nb}$ phages were enriched by 3 rounds of biopanning selection on microtiter plates (Nunc) coated with recombinant MMR. Individual colonies were screened in ELISA for antigen recognition and sequenced. The Nb gene of clone HS45, which recognizes both mouse and human NRP1, was recloned into the vector pHEN6 to encode a C-terminal His6 tag for purification purposes. Prior to use in cell culture, the Nbs were incubated 1 to 2 times with Pierce High Capacity Endotoxin Removal Resin (Thermo Fisher Scientific) until the residual endotoxin level was under the detection limit of the Kinetic Chromogenic LAL Assay (Lonza). Equal amounts of the nontargeted Nb BCII10 (50) were applied as negative control (Nb-ctrl).

Establishment of acquired cancer cell resistance to oncogene-targeted inhibitors. In order to establish melanoma cells resistant to PLX-4720, we treated naive A375 and naive SK-MEL-28 cells with escalating concentrations of the drug (starting from $125 \mathrm{nM}$ and progressively increasing the concentration up to $2 \mu \mathrm{M}$ ) until they acquired the ability to grow in the presence of PLX-4720 at the same rate as parental cells in the absence of the drug. Similarly, we treated BT474 and SKBR3 breast cancer cells with escalating concentrations of lapatinib (starting from $15 \mathrm{nM}$ and progressively increasing the concentration up to $250 \mathrm{nM}$ for BT474 and up to $120 \mathrm{nM}$ for SKBR3 cells) until they acquired the ability to grow at the same rate as parental cells in the absence of the drug.

Transient cell transfection with cDNA and siRNA. cDNA- and siRNA-expressing constructs were transiently transfected in mammalian cells with Lipofectamine 2000 (Life Technologies) according to the manufacturer's instructions. For reverse cell transfection experiments, the cells were transfected immediately after detachment. Briefly, the cells were detached with trypsin and counted. In the meantime, a mix of siRNA or cDNA, Opti-MEM medium, and lipofectamine was prepared (according to the manufacturer's instructions) and incubated at room temperature for 20 minutes. The transfection mix was dispensed first in a 96-well multiwell plate, immediately followed by the cell suspension $\left(1 \times 10^{3}-4 \times 10^{3}\right.$ per well, depending on cell line), in quadruplicate points. The transfected cells were directly analyzed in the wells 72-96 hours after transfection using cell viability assays.

Gene transfer. p27-expressing cDNA constructs were obtained from Addgene (plasmids 15192 and 14049). Lentiviral-mediated gene transfer in mammalian cells was accomplished as previously described (51). Nonreplicating viral particles containing transfer plasmids (or empty vector noncoding plasmids as control) or targeted shRNAs were pro- duced in HEK-293T packaging cells by cotransfection with the calcium phosphate precipitation method. Target cells were then incubated with the conditioned media derived from transfected $293 \mathrm{~T}$ cells, in the presence of polybrene $8 \mu \mathrm{g} / \mathrm{ml}$, for $8-12$ hours. This method ensured stable gene transfer with very high efficiency (over 95\% transgene-positive cells, as determined by immunostaining) without the need to select individual cell clones. In addition, to rule out variability of biological responses, at least 3 independent batches for each construct that was used to transduce cells were tested. In order to induce NRP1 expression, lentiviral-mediated gene transfer was applied by transducing vectors containing human NRP1 cDNA subcloned into the puromycinselectable NSPI lentiviral transfer plasmid (provided by Gera Neufeld, Israel Institute of Technology, Haifa, Israel).

Gene expression knockdown by RNA interference. NRP1 expression was silenced in tumor cells by transfecting targeted siRNA sequences (with Lipofectamine 2000, Invitrogen) or by transducing cells with shRNA-expressing lentiviral constructs (to achieve stable knockdown). EGFR expression was silenced by the following siRNA: GCAGUGACUUUCUCAGCAA and GCAGUCUUAUCUAACUAUGAU; SOX2 expression was silenced using a pool of 2 different siRNA: GGUUGACACCGUUGGUAAU and UGCCGAGAAUCCAUGUAUA; VEGFA was knocked down by means of 2 different siRNA: AUGUGAAUGCAGACCAAAG and AUGUGAAUGCAGACCAAAG. Two different sequences of chemically synthesized siRNA targeting NRP1 were used as a pool: (a) GAGAGGUCCUGAAUGUUCC, (b) AACACCUAGUGGAGUGAUA. To achieve long-term expression, the NRP1-targeting sequence above (a) was inserted in the lentiviral transfer plasmid pCCLsin.PPT.hPGK. GFP.Wpre in the frame of a sequence driving the transcription of a short-hairpin RNA under control of the $\mathrm{H} 1$ promoter, as previously reported (52). Control shRNA (shC) was generated by introducing 4 base substitutions in the NRP1 targeting sequence (GATAGGTCATGACTGCCC). We silenced NRP1 expression by means of a puromycinselectable lentiviral construct TRCNOOO0323055, provided by SigmaAldrich; plkO vector was used as control. SOX10 expression was stably knocked down by means of a doxycycline-inducible shRNA construct, provided by Liqin Wang (The Netherlands Cancer Institute [NKI], Amsterdam, The Netherlands), or by a second construct (b, above) expressing an independent shRNA sequence in a constitutive manner (TRCN0000018984, Sigma-Aldrich). cJUN and GIPC were silenced by means of 2 different shRNAs purchased from SigmaAldrich (cJUN: TRCNO000039591-1 and TRCNO000355647-2; GIPC: TRCNO000289267-1 and TRCNO000036769-2). Pre-miRNA (miRNA precursor) hsa-miR-338-3p, provided by Ambion (catalog PM10716), was transfected with Lipofectamine 2000 (Invitrogen).

RNA isolation and real-time PCR. Total RNA from tumor cell lines or tissues was isolated with the RNeasy Mini Kit (Qiagen) according to the manufacturer's instructions. cDNA preparation was done according to standard procedures, using M-MLV Reverse Transcriptase (Promega) and oligo-dT primers (Promega). Gene expression was measured using the following Taqman gene-specific probes from Thermo Fisher Scientific:NRP1 (Hs00826128_m1), EGFR (Hs00193306_m1), and the housekeepers GAPDH (Hs04420632_g1) and $\beta$-actin (Hs99999903_m1). The expression of the following genes was assessed by means of SYBR Green-specific primer pairs: SOX1O (Bio-Rad, qHsaCED0038654), p27 (CTGAGGACACGCATTTGGT, GGGGAACCGTCTGAAACAT), IGF1R (TACGTGAAGATCCGCCATTC, TCGAGGACGTAGAAGGAGTAAT), SOX2 (TGGGTTCGGTGGTCAAGTC， GCTCTGG- 
TAGTGCTGGGACA), cJUN (Biorad, qHsaCED0018770), VEGFA (TCCGGGTTTTATCCCTCTTC, ССTCTTTCTGCTGGTTTCCA), and the housekeeper genes GAPDH (GAAGGTGAAGGTCGGAGTC, GAAGATGGTGATGGGATTTC) and $\beta$-actin (CACTCTTCCAGCCTTCCTTC, GTACAGGTCTTTGCGGATGT). Real-time PCR analysis was performed using the Applied Biosystems 7900HT Fast RealTime PCR System. Total miRNAs were isolated with the miRNeasy Mini Kit (Qiagen) according to the manufacturer's instructions. miRNA expression was measured using Taqman gene-specific probes for hsamiR-338-3p (catalog 002252) and the housekeeper transcript RNU48 (catalog 001006) (both from Thermo Fisher Scientific).

Cell proliferation analysis. Tumor cells were seeded in multiple 96-well plates at an initial density of $1.5 \times 10^{3}-3 \times 10^{3}$ cells per well (depending on the cell line), and subsequently grown in complete medium. At every time point, one multiwell dish was fixed with $11 \%$ glutaraldehyde, stained with crystal violet, and the absorbance was read using a standard colorimetric system at $595 \mathrm{~nm}$.

Cell viability assays. Transduced tumor cells were seeded in 96-well plastic culture plates at an initial density of $1 \times 10^{3}-2 \times 10^{3}$ cells per well (depending on the cell line) in the presence of the indicated drugs or vehicle (DMSO). At 72 hours after cell seeding, the medium was changed and cell viability was assessed by CellTiter-Glo Luminescent Cell Viability Assay (Promega) according to the manufacturer's instructions. The number of viable cells was directly proportional to the luminescence signal recorded.

Western blotting analysis. Total protein extracts were obtained by cell lysis in LB-SDS buffer (25\% Tris $\mathrm{HCl} 0.5 \mathrm{M} \mathrm{pH} 6.8,25 \%$ SDS 10\%, $50 \% \mathrm{ddH}_{2} \mathrm{O}$ ). Protein concentrations were quantified by BCA Protein Assay kit (Thermo Fisher Scientific) and equal amounts of total cellular lysates were separated by SDS-PAGE and transferred to nitrocellulose membranes, which were blocked by incubation in $10 \%$ BSA. The membranes were incubated in the specific primary antibody properly diluted and then in the appropriate peroxidase-conjugated secondary antibody (Promega). Signal detection was done by ECL (Amersham Biosciences, GE Healthcare). Band densitometry analysis was done with Image J software (NIH) and myImageAnalysis (Thermo Fisher Scientific).

Phosphoproteomic array. The phosphorylation of multiple receptor tyrosine kinases was detected using the Proteome Profiler Array Kit ARY001 (R\&D Systems) according to manufacturer's protocols.

Statistics. Statistical significance was assessed using the 2-tailed Student's $t$ test or 2-way ANOVA test, with Bonferroni's correction applied when appropriate, as indicated in the figure legends. Error bars represent the standard deviation. All experiments were repeated at least 3 times (biological replicates). Figures show 1 representative experiment, reporting the average of the technical replicates. Asterisks indicated statistical significance according to this legend: ${ }^{*} P<$ $0.01 ;{ }^{* *} P<0.001 ;{ }^{* *} P<0.0001$.

Mouse experiments and study approval. Mouse handling and experiments followed international guidelines. The study was approved by the local ethical commission and by the Italian Ministry of Health. Specifically, $5 \times 10^{6} \mathrm{~A} 375$ or $2 \times 10^{6} \mathrm{EBC} 1$ tumor cells (resuspended in $200 \mu \mathrm{lBS}$ ) were injected subcutaneously in NOD/SCID female mice (Charles River Laboratories). Tumor volume was periodically measured with a digital caliper during 2 of 3 week-long experiments. Two-dimensional fluorescence imaging of red fluorescent proteinlabeled (RFP-labeled) tumor cells in vivo was performed with the IVIS Lumina II system (Perkin Elmer). Laser image acquisitions (excitation $570 \mathrm{~nm}$, emission $620 \mathrm{~nm}$ ) were done twice a week, analyzing previously shaved, anesthetized mice. Fluorescent signal in regions of interest was quantified as photon total flux, using Living Image software 4.3.1 (Perkin Elmer). At the end of the experiment, the mice were euthanized and tumor masses were lysed for genomic DNA analysis. For drug-treatment experiments, the mice were treated orally by gavage with PLX-4720 or JNJ-605 inhibitors (or with DMSO vehicle) diluted in a solution of $1 \%$ (hydroxypropyl)-methylcellulose (MilliporeSigma, catalog H7509) and 0.2\% Tween80 (MilliporeSigma, catalog P1754).

Genomic DNA analysis. Explanted tumor xenografts were immediately snap frozen and homogenized with Ultra Turrex T8. The genomic DNA was then extracted with the ReliaPrep Tissue gDNA Miniprep extraction system (Promega) and analyzed using the Applied Biosystems 7900HT Fast Real-Time PCR System with the following primers: U6 (CCCTCGTTGACCGAATCAC, CACCGTGGGCTTGTACTC), tRPF (GCAGCAAAGCCTTCATCAAC, CTGCTTCGTATGTGGTGATTCT), and puromycin resistance cassette (plkO) (GCGCAGCAACAGATGGAA, CAGACCCTTGCCCTGGT).

\section{Author contributions}

SR and LT designed and performed experiments, analyzed the data, and contributed to manuscript writing. GC and CB executed experiments and data analyses. SB and CI contributed to data collection and analysis. JAVG, RB, FDN, and SG contributed to experimental design, data analysis, and manuscript writing.

\section{Acknowledgments}

We are grateful to Massimo Accardo for help with experiments in mouse models, to Barbara Martinoglio for help with real-time PCR analysis, to the lab of Guido Serini for help with endothelial cell experiments, and to Livio Trusolino and all Tamagnone lab members for advice and support. We are grateful to Lawrence Kwong (Chin laboratory, The University of Texas MD Anderson Cancer Center) for giving access to the RNA-Seq data set derived from melanoma samples (EGAD00001001306). This work was supported by grants from the Italian Association for Cancer Research (AIRC-IG 2017-19923 to LT and AIRC-IG 2017-20210 to SG) and the Fondazione Piemontese per la Ricerca sul Cancro (grants FPRC-5perMille-MIUR-2013 and FPRC-5perMille-Ministero Salute-2013 to LT). SB is supported by a doctoral grant from the Flemish Agency for Innovation by Science and Technology. JAVG is supported by Kom op tegen kanker, Stichting tegen Kanker, and the Fund for Scientific Research.

Address correspondence to: Luca Tamagnone, Candiolo Cancer Institute, IRCCS, Str. Prov. 142, 10060, Candiolo (TO), Italy. Phone: 39.011.9933204; Email: luca.tamagnone@ircc.it.
1. Torti D, Trusolino L. Oncogene addiction as a foundational rationale for targeted anti-cancer therapy: promises and perils. EMBO Mol Med. 2011;3(11):623-636.
2. Arteaga CL, Engelman JA. ERBB receptors: from oncogene discovery to basic science to mechanism-based cancer therapeutics. Cancer Cell. 2014;25(3):282-303.
3. Johnson DB, et al. Acquired BRAF inhibitor resistance: A multicenter meta-analysis of the spectrum and frequencies, clinical behaviour, and phenotypic associations of resistance mech- 
anisms. Eur J Cancer. 2015;51(18):2792-2799.

4. Camidge DR, Pao W, Sequist LV. Acquired resistance to TKIs in solid tumours: learning from lung cancer. Nat Rev Clin Oncol. 2014;11(8):473-481.

5. Fallahi-Sichani M, et al. Adaptive resistance of melanoma cells to RAF inhibition via reversible induction of a slowly dividing de-differentiated state. Mol Syst Biol. 2017;13(1):905.

6. Wilson TR, et al. Widespread potential for growthfactor-driven resistance to anticancer kinase inhibitors. Nature. 2012;487(7408):505-509.

7. Rizzolio S, Tamagnone L. Multifaceted role of neuropilins in cancer. Curr Med Chem. 2011;18(23):3563-3575.

8. Guttmann-Raviv N, Kessler O, Shraga-Heled N Lange T, Herzog Y, Neufeld G. The neuropilins and their role in tumorigenesis and tumor progression. Cancer Lett. 2006;231(1):1-11.

9. Rizzolio S, et al. Neuropilin-1-dependent regulation of EGF-receptor signaling. Cancer Res. 2012;72(22):5801-5811.

10. Li L, et al. Neuropilin-1 is associated with clinicopathology of gastric cancer and contributes to cell proliferation and migration as multifunctional co-receptors. J Exp Clin Cancer Res. 2016;35:16.

11. Rizzolio S, Tamagnone L. Neuropilins as signaling hubs, controlling tyrosine kinases and other cell surface receptors. In: Neufeld G, Kessler O, eds. The neuropilins: role and function in health and disease. New York, NY: Springer International Publishing; 2017:23-39.

12. Prud'homme GJ, Glinka Y. Neuropilins are multifunctional coreceptors involved in tumor initiation, growth, metastasis and immunity. Oncotarget. 2012;3(9):921-939.

13. Ding H, et al. Expression and regulation of neuropilin-1 in human astrocytomas. Int J Cancer. 2000;88(4):584-592.

14. Lichtenberger BM, Tan PK, Niederleithner H, Ferrara N, Petzelbauer P, Sibilia M. Autocrine VEGF signaling synergizes with EGFR in tumor cells to promote epithelial cancer development. Cell. 2010;140(2):268-279.

15. Liu W, Wu T, Dong X, Zeng YA. Neuropilin-1 is upregulated by Wnt/ $\beta$-catenin signaling and is important for mammary stem cells. Sci Rep. 2017;7(1):10941.

16. Peng Y, Liu YM, Li LC, Wang LL, Wu XL. MicroRNA-338 inhibits growth, invasion and metastasis of gastric cancer by targeting NRP1 expression. PLoS One. 2014;9(4):e94422.

17. Sun C, et al. Reversible and adaptive resistance to BRAF(V600E) inhibition in melanoma. Nature. 2014;508(7494):118-122.

18. Barr MP, et al. Vascular endothelial growth factor is an autocrine growth factor, signaling through neuropilin-1 in non-small cell lung cancer. $\mathrm{Mol}$ Cancer. 2015;14:45.

19. Hamerlik P, et al. Autocrine VEGF-VEGFR2Neuropilin-1 signaling promotes glioma stemlike cell viability and tumor growth. J Exp Med. 2012;209(3):507-520.
20. Grun D, Adhikary G, Eckert RL. VEGF-A acts via neuropilin-1 to enhance epidermal cancer stem cell survival and formation of aggressive and highly vascularized tumors. Oncogene. 2016;35(33):4379-4387.

21. Beck B, et al. A vascular niche and a VEGF-Nrp1 loop regulate the initiation and stemness of skin tumours. Nature. 2011;478(7369):399-403.

22. Goel HL, Mercurio AM. VEGF targets the tumour cell. Nat Rev Cancer. 2013;13(12):871-882.

23. Gokey NG, Srinivasan R, Lopez-Anido C, Krueger C, Svaren J. Developmental regulation of microRNA expression in Schwann cells. Mol Cell Biol. 2012;32(2):558-568.

24. Liu C, Wang Z, Wang Y, Gu W. MiR-338 suppresses the growth and metastasis of OSCC cells by targeting NRP1. Mol Cell Biochem. 2015;398(1-2):115-122.

25. Apicella M, et al. Dual MET/EGFR therapy leads to complete response and resistance prevention in a MET-amplified gastroesophageal xenopatient cohort. Oncogene. 2017;36(9):1200-1210.

26. Rexer BN, Arteaga CL. Intrinsic and acquired resistance to HER2-targeted therapies in HER2 gene-amplified breast cancer: mechanisms and clinical implications. Crit Rev Oncog. 2012;17(1):1-16

27. Merry CR, McMahon S, Thompson CL, Miskimen KL, Harris LN, Khalil AM. Integrative transcriptome-wide analyses reveal critical HER2-regulated mRNAs and lincRNAs in HER2+ breast cancer. Breast Cancer Res Treat. 2015;150(2):321-334.

28. Yoshida A, et al. VEGF-A/NRP1 stimulates GIPC1 and Syx complex formation to promote RhoA activation and proliferation in skin cancer cells. Biol Open. 2015;4(9):1063-1076.

29. Fang Y, et al. A new tumour suppression mechanism by p27Kip1: EGFR down-regulation mediated by JNK/c-Jun pathway inhibition. Biochem J. 2014;463(3):383-392.

30. Wey JS, et al. Overexpression of neuropilin-1 promotes constitutive MAPK signalling and chemoresistance in pancreatic cancer cells. Br J Cancer. 2005;93(2):233-241.

31. Chou YT, et al. The emerging role of SOX 2 in cell proliferation and survival and its crosstalk with oncogenic signaling in lung cancer. Stem Cells. 2013;31(12):2607-2619.

32. Hu Q, et al. The EGF receptor-sox2-EGF receptor feedback loop positively regulates the selfrenewal of neural precursor cells. Stem Cells. 2010;28(2):279-286.

33. Wuebben EL, Rizzino A. The dark side of SOX2: cancer - a comprehensive overview. Oncotarget. 2017;8(27):44917-44943.

34. Scheidegger KJ, Du J, Delafontaine P. Distinct and common pathways in the regulation of insulin-like growth factor-1 receptor gene expression by angiotensin II and basic fibroblast growth factor. JBiol Chem. 1999;274(6):3522-3530.

35. Christopoulos PF, Msaouel P, Koutsilieris M. The role of the insulin-like growth factor-1 system in breast cancer. Mol Cancer. 2015;14:43.

36. Jarvis A, et al. Small molecule inhibitors of the neuropilin-1 vascular endothelial growth factor A (VEGF-A) interaction. J Med Chem. 2010;53(5):2215-2226

37. Schoonooghe S, et al. Novel applications of nanobodies for in vivo bio-imaging of inflamed tissues in inflammatory diseases and cancer. Immunobiology. 2012;217(12):1266-1272.

38. Van Allen EM, et al. The genetic landscape of clinical resistance to RAF inhibition in metastatic melanoma. Cancer Discov. 2014;4(1):94-109.

39. Manzano JL, et al. Resistant mechanisms to BRAF inhibitors in melanoma. Ann Transl Med. 2016;4(12):237.

40. Wang J, et al. Epigenetic changes of EGFR have an important role in BRAF inhibitor-resistant cutaneous melanomas. J Invest Dermatol. 2015;135(2):532-541.

41. Akagi M, et al. Induction of neuropilin-1 and vascular endothelial growth factor by epidermal growth factor in human gastric cancer cells. $\mathrm{Br} \mathrm{J}$ Cancer. 2003;88(5):796-802.

42. Parikh AA, et al. Neuropilin-1 in human colon cancer: expression, regulation, and role in induction of angiogenesis. Am J Pathol. 2004;164(6):2139-2151.

43. Pang HB, et al. An endocytosis pathway initiated through neuropilin-1 and regulated by nutrient availability. Nat Commun. 2014;5:4904.

44. Boumahdi S, et al. SOX2 controls tumour initiation and cancer stem-cell functions in squamous-cell carcinoma. Nature. 2014;511(7508):246-250.

45. McDermott MSJ, et al. Dual inhibition of IGF1R and ER enhances response to trastuzumab in HER2 positive breast cancer cells. Int JOncol. 2017;50(6):2221-2228.

46. Cai H, Reed RR. Cloning and characterization of neuropilin-1-interacting protein: a PSD-95/Dlg/ ZO-1 domain-containing protein that interacts with the cytoplasmic domain of neuropilin-1. J Neurosci. 1999;19(15):6519-6527.

47. Casazza A, et al. Impeding macrophage entry into hypoxic tumor areas by Sema3A/Nrp1 signaling blockade inhibits angiogenesis and restores antitumor immunity. Cancer Cell. 2013;24(6):695-709.

48. Overacre-Delgoffe AE, et al. Interferon- $\gamma$ drives Treg fragility to promote anti-tumor immunity. Cell. 2017;169(6):1130-1141.e11.

49. Kwong LN, et al. Co-clinical assessment identifies patterns of BRAF inhibitor resistance in melanoma. JClin Invest. 2015;125(4):1459-1470.

50. Conrath KE, et al. Beta-lactamase inhibitors derived from single-domain antibody fragments elicited in the camelidae. Antimicrob Agents Chemother. 2001;45(10):2807-2812.

51. Follenzi A, Naldini L. HIV-based vectors. Preparation and use. Methods Mol Med. 2002;69:259-274.

52. Corso S, Migliore C, Ghiso E, De Rosa G, Comoglio PM, Giordano S. Silencing the MET oncogene leads to regression of experimental tumors and metastases. Oncogene. 2008;27(5):684-693. 